\title{
THE APRIL MEETING IN PHILADELPHIA
}

The four hundred forty-sixth meeting of the American Mathematical Society was held at The University of Pennsylvania, Philadelphia, Pennsylvania, on Friday and Saturday, April 29-30. The attendance was about three hundred, including the following two hundred forty-three members of the Society.

C. R. Adams, R. B. Adams, E. J. Akutowicz, A. A. Albert, C. B. Allendoerfer, Warren Ambrose, R. D. Anderson, S. I. Askovitz, Iacopo Barsotti, F. D. Bateman, P. T. Bateman, F. W. Beal, F. P. Beer, E. G. Begle, Stefan Bergman, Lipman Bers, R. J. Bickel, Garrett Birkhoff, A. L. Blakers, W. A. Blankinship, J. H. Blau, R. P. Boas, Enrico Bompiani, W. M. Boothby, D. G. Bourgin, R. C. Briant, R. C. Campbell, P. A. Caris, K. Chandrasekharan, S. S. Chern, P. L. Chessin, Randolph Church, Edmund Churchill, J. A. Clarkson, I. S. Cohen, L. W. Cohen, R. H. Cole, Damian Connelly, A. H. Copeland, Natalie Coplan, G. F. Cramer, C. W. Crouse, A. B. Cunningham, H. B. Curry, J. H. Curtiss, D. A. Darling, M. M. Day, C. S. Dias, E. M. DiDomenica, M. P. Dolciani, J. L. Doob, C. H. Dowker, Y. N. Dowker, R. H. Downing, L. A. Dragonette, Aryeh Dvoretzky, J. J. Eachus, Samuel Eilenberg, Bernard Epstein, Paul Erdös, G. C. Evans, O. J. Ferrell, William Feller, N. J. Fine, C. D. Firestone, E. E. Floyd, R. M. Foster, R. H. Fox, Gerald Freilich, Orrin Frink, David Gale, G. N. Garrison, J. B. Giever, W. M. Gilbert, B. P. Gill, O. E. Glenn, H. E. Goheen, H. H. Goldstine, H. H. Goode, M. J. Gottlieb, W. H. Gottschalk, Arthur Grad, Emil Grosswald, C. C. Grove, V. H. Haefeli, M. R. Hafner, Harish-Chandra, K. E. Hazard, G. A. Hedlund, A. E. Heins, M. H. Heins, Alex Heller, Erik Hemmingsen, Coleman Herpel, A. D. Hestenes, T. H. Hildebrandt, Joseph Hilsenrath, T. R. Hollcroft, E. M. Hull, Witold Hurewicz, L. C. Hutchinson, K. S. Jacoby, M. L. Juncosa, Shizuo Kakutani, Hyman Kamel, Irving Kaplansky, Gilbert Kaskey, W. H. Keen, M. E. Kellar, D. E. Kibbey, Fred Kiokemeister, J. R. Kline, T. L. Koehler, D. D. Kosambi, H. W. Kuhn, V. V. Latshaw, Solomon Lefschetz, Joseph Lehner, Marguerite Lehr, R. A. Leibler, R. B. Leipnik, George Lewis, M. A. Lipschutz, S. R. Lipsey, Charles Loewner, E. R. Lorch, Lee Lorch, H. M. Lufkin, D. L. McDonough, E. A. McDougle, J. A. McKee, G. W. Mackey, Murray Mannos, M. H. Martin, W. T. Martin, W. S. Massey, A. E. Meder, L. I. Mishoe, E. E. Moise, Deane Montgomery, T. W. Moore, K. A. Morgan, C. R. Morris, Marston Morse, G. D. Mostow, T. S. Motzkin, W. R. Murray, A. C. Neison, Paolo Nesbeda, Morris Newman, C. V. Newsom, R. Z. Norman, C. O. Oakley, Paul Olum, F. W. Owens, H. B. Owens, J. C. Oxtoby, J. S. Oxtoby, O. O. Pardee, A. J. Penico, F. W. Perkins, I. D. Peters, R. P. Peterson, H. P. Pettit, Joel Pitcairn, Everett Pitcher, Pasquale Porcelli, W. W. Proctor, M. H. Protter, W. T. Puckett, Gustave Rabson, Hans Rademacher, John Raleigh, K. G. Ramanathan, H. E. Rauch, A. G. Rawling, G. E. Raynor, C. J. Rees, K. W. Reidemeister, Eric Reissner, Russell Remage, M. M. Resnikoff, C. N. Reynolds, J. E. Robinson, S. L. Robinson, P. C. Rosenbloom, J. B. Rosser, C. W. Saalfrank, Arthur Sard, S. W. Saunders, A. B. Schacknow, A. T. Schafer, R. D. Schafer, Albert Schild, Pincus Schub, Abraham Schwartz, G. E. Schweigert, D. B. Shaffer, I. M. Sheffer, Seymour Sherman, S. P. Shugert, Edward Silverman, M. L. Slater, M H. Slud, Andrew Sobczyk, C. F. Stephens, M. H. Stone, W. J. Strange, A. H. Taub, W. R. Thickstun, J. M. Thomas, M. L. Tomber, C. B. Tompkins, D. L. Thomsen, C. A Truesdell, A. W. Tucker, W. J. Turanski, J. R. Van Andel, J. L. Vanderslice, H. E: 
Vansant, A. H. Van Tuyl, Oswald Veblen, Sylvan Wallach, J. L. Walsh, J. B. Walton, W. R. Wasow, G. C. Webber, M. A. Weber, J. V. Wehausen, Alexander Weinstein, D. W. Western, P. M. Whitman, A. M. Whitney, Hassler Whitney, Albert Wilansky, W. G. Wolfgang, Y. K. Wong, Marcella Yarosheski, G. S. Young, J. W. Young, L. C. Young, Oscar Zariski, P. W. Zettler-Seidel.

On Friday afternoon Professor G. W. Mackey of Harvard University gave an address on Functions on locally compact groups. Professor M. H. Stone presided.

President J. L. Walsh presided at the general session Saturday morning at which an address was given by Professor K. W. Reidemeister of the University of Marburg and Institute for Advanced Study on The complexes of a group. Following the address, President Walsh presented embossed sets of resolutions of appreciation from the Board of Trustees and the Council of the American Mathematical Society to Professor Bennington P. Gill, retiring Treasurer of the Society. The resolutions adopted by the Council appear in the report of the 1948 Annual Meeting. Professor Gill thanked the Board of Trustees and the Council and spoke briefly on the expanding activities of the Society during his eleven years' service.

President Walsh introduced Professor D. G. Bourgin who presented resolutions of thanks to the President, the Mathematics Department, and the Museum officials of the University of Pennsylvania for their excellent arrangements and cordial hospitality.

On Friday afternoon there were two sections, one for papers in Statistics and Applied Mathematics in which Professor Eric Reissner presided, and one for papers in Analysis, Geometry, and Topology in which Professor Samuel Eilenberg presided. On Saturday afternoon there were two sections, one for papers in Analysis in which Professor C. R. Adams presided, and one for papers in Algebra and Topology in which Professor A. H. Copeland presided.

President and Mrs. Harold E. Stassen of the University of Pennsylvania received the mathematicians and their guests at a tea in the Middle American Hall of the University Museum at 4:30 P.M. on Friday.

The Council met at 2:00 P.M. on April 30 .

The Secretary announced the election of the following ninety-five persons to ordinary membership in the Society:

Mr. Allan Henry Albert, University of Michigan;

Miss Linda Allegri, Hunter College High School;

Mr. Raymond William Allen, St. Louis University;

Mr. Richard Lewis Arnowitt, Cambridge, Mass.;

Professor Antonio Astorga, Reserve Officers Preparatory Center, Rio de Janeiro, Brazil; 
Mr. Robert Baer, Roosevelt College, Chicago, Illinois;

Mr. David Campbell Barton, Roberts Junior College, North Chili, N. Y.;

Mr. Mohammedali Abdul Ameer Bassam, University of Texas;

Mr. Vernon Nelson Behrns, University of Buffalo;

Mr. Fred George Berghoefer, Chicago, Ill.;

Helen Virena Betz (Mrs. G. P.), Northwestern University;

Miss Lollie Belle Bienvenu, Louisiana State University and Agricultural and Mechanical College;

Professor John Henry Binney, Agricultural and Mechanical College of Texas;

Mr. Jacob Borsuk, Evans Signal Laboratory, Belmar, N. J.;

Mr. Joseph Edward Bosshart, Cleveland, Ohio;

Dr. John R. Bowman, Mellon Institute;

Mr. Frank Herbert Brownell III, Princeton University;

Mr. George Hench Butcher, Howard University;

Mr. William Albert Catenaro, University of Oklahoma;

Mr. Philip Cooperman, New York University;

Miss Grace Virginia Crenshaw, Danville, Va.;

Mr. Fred T. Daly, Ithaca, N. Y.;

Dr. Walter Lorraine Deemer, Lt. Col., U. S. Air Forces, University of North Carolina;

Mr. George Henry DeMers, Urbana, Ill.;

Mr. Bernard Emile Dethier, Nazareth College;

Professor Louis E. Diamond, Oklahoma City University;

Mr. John Allen Doggett, Howard University;

Mr. Robert Leonard Evans, University of Minnesota;

Mr. Frank David Faulkner, University of Michigan;

Professor James Kern Feibleman, Tulane University of Louisiana;

Mr. Marvin Julian Forray, Polytechnic Institute of Brooklyn;

Mr. Jerome Bernard Freier, New York University;

Mr. William Barkley Fritz, Ballistic Research Laboratory, Aberdeen Proving Ground, Md.;

Mr. Robert Arthur Fuchs, University of California;

Dr. Boris Garfinkel, Ballistic Research Laboratory, Aberdeen Proving Ground, Md.;

Mr. Dal Charles Gerneth, Sherman, Texas;

Mr. Gene Heiden Gleissner, College of Pharmacy, Columbia University;

Professor Frederick George Graff, Oberlin College;

Mr. Oliver Alfred Gross, Rand Corporation, Santa Monica, Calif.;

Miss Rose Ann Grundman, University of Arizona;

Mr. Benjamin Franklin Handy, Jr., National Bureau of Standards, Washington, D. C.;

Mr. Frank Sylvester Hawthorne, Hofstra College;

Dr. Alberta H. Henry, Brooklyn College;

Professor Mildred Elizabeth Hull, Wilkes College;

Professor Lamek Hulthen, Royal Institute of Technology, Stockholm, Sweden;

Mr. William Elliott Jenner, University of British Columbia;

Commander Walter Hughes Keen, U. S. Navy, Princeton University;

Mr. Ralph Craig Kennedy, National Broacasting Company, New York, N. Y.;

Miss Evelyn Kendrick Kinney, University of Illinois;

Mr. Robert Elmer Kruncklin, American Viscose Corporation, Philadelphia, Pa.;

Mr. Ke-chan Kuo, University of Illinois;

Mr. Paul Gustav Kvick, Columbia University;

Dr. Robert Steven Ledley, Columbia University; 
Mr. James A. Lemon, Fenn College;

Miss Dinah G. Levine, Polytechnic Institute of Brooklyn;

Mr. Leo Meyer Levine, Naval Shipyard, Brooklyn, N. Y.;

Dr. Mark Lotkin, Ballistic Research Laboratory, Aberdeen Proving Ground, Md.;

Miss Hilda Marie Lucy, Howard University;

Mr. Robert Kinsey McConnell, Jr., New York University;

Dr. Clifford Joseph Maloney, Research and Development Department, Camp Detrick, Frederick, Md.;

Mr. John Maurice Marr, University of Missouri;

Mr. Norman M. Martin, University of Illinois;

Dr. John George Meiler, Coos Bay Lumber Company, Coos Bay, Ore.;

Professor John Graham Millar, University of Alberta, Calgary, Alta., Canada;

Mr. Paul Dixon Minton, University of North Carolina;

Miss Mabel D. Montgomery, University of Buffalo;

Mr. August Carl Nelson, Jr., University of Delaware;

Mr. Cesar Dacorso Netto, Rio de Janeiro, Brazil;

Professor Helen Olney, Hiram College, Hiram, Ohio;

Professor Richard Robert Otter, University of Notre Dame;

Mr. Bertram Samuel Page, Brotherton Library, University of Leeds;

Mr. Stephen Tyler Paine, Institute for Numerical Analysis, National Bureau of Standards, University of California at Los Angeles;

Dr. Otway O'Meara Pardee, Syracuse University;

Mr. Jerome Kenneth Percus, Columbia University;

Professor Iland Dee Peters, West Virginia University;

Mr. Aihud Pevsner, New York, N. Y.;

Mr. George Walter Reitwiesner, Ballistic Research Laboratory, Aberdeen Proving Ground, Md.;

Professor Antonio Romero-Juarez, University of Mexico;

Mr. Nicholas John Rose, Stevens Institute of Technology;

Mr. Paul Talbot Rotter, Mutual Benefit Life Insurance Company, Newark, N. J.;

Mr. Theodore Rubin, Fairchild Engine and Airplane Corp., Oak Ridge, Tenn.;

Mr. Edward Lee Schlain, Moore School of Electrical Engineering, University of Pennsylvania;

Mr. David Jerome Smith, University of Notre Dame;

Mr. Harry Smith, Jr., University of Delaware;

Mr. James Edward Snover, Sampson College, Sampson, N. Y.;

Mr. Anson Donald Solem, Naval Ordnance Laboratory, White Oak, Silver Spring, Md.;

Mr. Nathaniel Hawthorne Stevens, Franklin School, New York, N. Y.;

Mr. Joseph A. Sullivan, Indiana University;

Professor Henry William Syer, Boston University;

Mr. Gerald Luther Thompson, University of Michigan;

Mr. William Thomas Tutte, University of Toronto;

Mr. Robert Ullman, Polytechnic Institute of Brooklyn;

Mr. James Hatton Wahab, University of North Carolina;

Mr. William Guinn Wolfgang, Altoona Center, Pennsylvania State College;

Dr. Albert Wolinsky, New York University.

It was reported that the following twenty-two persons had been elected to membership on nomination of institutional members as indicated: 
Brown University: Mr. Edward Forrest Moore;

Harvard University: Messrs. Seymour Hayden, Henry Helson, George Graham Murray, Henry Gardner Rogers, William J. Turanski, Neal Thomas Watson, Harold Weintraub, John Wermer, and Vidar Michael Wolontis;

Haverford College: Mr. Cloyd Marvin;

Institute for Advanced Study: Dr. Verena Huber Haefeli, Professor Kazimierz Kuratowski, Dr. Kurt Mahler, and Professor Andrzej Mostowski;

University of Minnesota: Mr. Philip George Kirmser;

Pennsylvania State College: Messrs. Winton Laubach and Richard Wesley Wonn; Purdue University: Messrs. James Dawson McKnight, Jr., Bill VanTrump Ritchie, Eugene Usdin;

University of Rochester: Mr. Arthur Edward Danese.

The Secretary announced that the following had been admitted to the Society in accordance with reciprocity agreements with various mathematical organizations: London Mathematical Society: Professor Joseph Langley Burchnall, Durham University; Dr. Mary Lucy Cartwright, Girton College, Cambridge University; Professor John Kronsbein, Evansville College, Evansville, Ind.; Dr. Samuel Verblunsky, Queen's University, Belfast, Northern Ireland; Dr. Thomas James Willmore, Durham Colleges, University of Durham; Société Mathématique de France: Mr. Raymond George Ayoub, McGill University; Professor Georges Bourion, University of Algiers; Dr. Ion Carstoiu, National Center of Scientific Research, Paris; Professor Pierre Jacques Lelong, University of Lille; Swiss Mathematical Society: Dr. Harry Ernest Rauch, Rutgers University; Wiskundig Genootschap te Amsterdam: Professor Hans Freudenthal, University of Utrecht.

The following actions taken by mail vote of the Council were reported: election of Professors Samuel Eilenberg, T. H. Hildebrandt, Saunders MacLane and W. T. Martin as members of the Executive Committee of the Council, the first two to serve for a term of one year beginning January 1,1949, and the second two for a term of two years; acceptance of statement of policy in connection with papers submitted to the Transactions; acceptance of a manuscript by Professors A. C. Schaeffer and D. C. Spencer, entitled Coefficient regions of schlicht functions, for publication in the Colloquium Series; authorization of the appointment of a committee to organize an experiment in the reproduction of research by methods other than letter press; acceptance of a manuscript by Professor J. L. Walsh, entitled The location of critical points, for publication in the Colloquium Series.

The following appointments of representatives of the Society were reported: Professor J. L. Walsh at Mid-Century Convocation and inauguration of James Rhyne Killian, Jr., as President of Massachusetts Institute of Technology on March 31-April 2, 1949; Pro- 
fessor C. O. Oakley at Fifty-third Annual Meeting of the American Academy of Political and Social Science on April 8-9, 1949; Professor Gillie A. Larew at the inauguration of Walter Stephenson Newman as President of Virginia Polytechnic Institute on April 18, 1949; Professor A. E. Landry at the inauguration of Rev. Hunter Guthrie as President of Georgetown University on April 30-May 1, 1949; Professor S. S. Cairns at the inauguration of Delyte Wesley Morris as President of Southern Illinois University on May 5, 1949; Professor D. C. Lewis, Jr., at the inauguration of Otto Frederick Kraushaar as President of Goucher College on May 6-7, 1949; Professor Lincoln $\mathrm{LaPaz}$ at the inauguration of Thomas Lafayette Popejoy as President of the University of New Mexico on June 4, 1949.

The following additional appointments by the President were reported: Professor R. W. Brink as a member for the period 1949-1951 and Professor C. B. Allendoerfer as Chairman for 1949 of the Committee on Places of Meetings (committee now consists of Professors C. B. Allendoerfer, Chairman, R. W. Brink, and R. L. Wilder); Professors William Feller (Chairman), G. A. Hedlund, and M. H. Heins as a Committee to Select Gibbs Lecturers for 1950 and 1951; Professors T. H. Hildebrandt (Chairman), S. S. Cairns, L. M. Graves, J. W. Green, and W. T. Martin as a Committee to Nominate Officers and Members of the Council for 1950; Professors A. J. Kempner (Chairman), J. R. Britton, H. M. Gehman, J. W. Green, C. A. Hutchinson, B. W. Jones, Claribel Kendall, and O. H. Rechard as a Committee on Arrangements for the 1949 Summer Meeting; Professor E. R. Lorch (Chairman), Miss Grace Bolton, Professors T. F. Cope, H. M. Gehman, B. P. Gill, T. R. Hollcroft, S. B. Littauer, and Walter Prenowitz as a Committee on Arrangements for the 1949 Annual Meeting; Professors B. P. Gill (Chairman), Samuel Eilenberg and P. A. Smith as a Committee to Study Special Methods of Reproducing Mathematical Research; Professors A. W. Tucker (Chairman), R. P. Boas, Samuel Eilenberg, J. R. Kline, R. E. Langer, and C. J. Rees as an Emergency Publication Committee; Professors T. F. Cope and B. P. Gill as auditors of the Society's accounts for 1949. The following items were reported for the information of the Council: appointment of Professor L. M. Graves as Chairman of the Editorial Committee for the International Congress (to replace Professor Salomon Bochner, resigned); appointment of Dean A. E. Meder as an additional member of the Financial Committee of the Congress; selection of Professors Nelson Dunford and M. H. Stone as Chairmen of the Mathematical Surveys and Mathematical Reviews Editorial Committees, respectively, for 1949; establishment of 
a reciprocity agreement between the Society and the Norwegian Mathematical Society; receipt of a letter of thanks from London Mathematical Society for a gift of journals and books; acceptance from Professor Norbert Wiener of invitation to deliver the Gibbs Lecture at the 1949 Annual Meeting; approval by the Committee on Applied Mathematics of sponsorship by the Society of the following: Conference on Applied Mathematics of the American Institute of Electrical Engineers in New York City on January 31, 1949; Heat Transfer and Fluid Mechanics Institute at the University of California in June, 1949; Conference on Fluid Dynamics at the University of Illinois in the spring of 1950.

On recommendation of the Organizing Committee of the International Congress, the Council authorized extending invitations for hour addresses to twenty-seven mathematicians instead of to twentyfour, the maximum number recommended by the Eisenhart Report adopted by the Council in 1937. It was also reported that Congress fees had been set at $\$ 15.00$ for full membership and $\$ 7.50$ for associate membership.

The Council voted to recommend to the Trustees that the subscription prices for Mathematical Reviews be increased to the following: list price, $\$ 20.00$ per volume; price to members of sponsoring organizations, $\$ 10.00$; price to reviewers, $\$ 5.00$.

It was reported that Professors B $\phi$ rge Jessen and F. B. Jones had been invited to deliver hour addresses at the 1949 Summer Meeting.

Recommendations were presented from the Emergency Publication Committee which had also been approved by the Committee on the Role of the Society in Mathematical Publication. The Council adopted the following recommendations, designed to relieve the Society's publication crisis:

(1) That the present green issues of the Bulletin be published as a separate new journal, to be known as the Proceedings of the American Mathematical Society. It is hoped that it may be possible to print the Bulletin (present green issues) by photo-offset process, with considerable saving to the Society. Until the question of individual membership dues is re-studied, members will receive both the Bulletin and the Proceedings.

(2) That a new medium of publication be created, as soon as possible, for longer papers and groups of cognate papers, this medium to be known as the Memoirs of the American Mathematical Society. The Memoirs are to be under the editorial control of the Transactions Editorial Committee, with the same standards as the Transactions. The Memoirs are to be a non-periodical but serial publication, in 
this respect like Mathematical Surveys and the Colloquium Publications, and are to be issued and sold in separate volumes. The Memoirs are to be reproduced by photo-offset from copy completely prepared by the authors, following instructions formulated in a manual, but not restricted in style. Because of the financial commitments involved, the question of the Memoirs was referred to the Board of Trustees for final action. If approved by the Board, it is hoped that the Memoirs may be started late in 1949 or early in 1950 .

The following items were referred to a special committee to be appointed by the President: the recommendation that the per page rate on which institutional membership dues are based be increased from $\$ 2.75$ to $\$ 5.00$; the suggestion that the terms of individual membership be reconsidered, in light of the changes in the Society's publication program suggested above; the question of the relation between the Society and institutional members which publish mathematical journals.

It was reported that the Policy Committee for Mathematics had been studying questions concerned with the possible establishment of a mathematical foundation for the purpose of securing financial support from industry and other interested groups, the recommendations to Selective Service in connection with students in all fields of academic endeavor, and an International Mathematical Union.

The Council, on recommendation of the Policy Committee, approved the by-laws and statutes of the International Union of Theoretical and Applied Mechanics and voted to continue the Society's representation on the United States Committee on Theoretical and Applied Mechanics, the group through which the United States has adhered to the Union, and to recommend to the Trustees that a contribution be made to this Committee.

The Council voted to schedule meetings as follows: November 2526, 1949, at Illinois Institute of Technology; southeastern meeting of 1950 at Oak Ridge Laboratories; spring eastern meeting of 1950 in Washington, D. C.

The Council voted not to make available to authors reprints of articles appearing in the Proceedings of Applied Mathematics Symposia but voted that one copy of the whole volume should be given to each author.

Abstracts of the papers read follow below. Papers whose abstract numbers are followed by the letter " $t$ " were read by title. Paper number 336 was read by Mr. Kuhn, paper number 339 by Mr. Porcelli, paper number 342 by Professor Rosenbloom, paper number 365 by Miss Whitney, and paper number 390 by Dr. Blakers. Mr. 
Szekeres was introduced by Professor T. R. Hollcroft, Mr. Moore by Professor Herbert Federer, and Mr. Shanks by Dr. A. H. Van Tuyl.

\section{Algebra ANd Theory of Numbers}

\section{6t. A. A. Albert: A theory of trace-admissible algebras.}

Power associative algebras have been defined by many sets of identities (such as those defining Jordan algebras, alternative algebras, right alternative algebras, and standard algebras) and have been studied successfully only by the use of a trace function. The author now takes a major step forward in the study of such algebras by the derivation of a complete structure theory of all trace-admissible power associative algebras whose radical is defined to be the maximal nilideal. Then semisimple algebras are direct sums of simple algebras, the attached algebra $A^{(+)}$is always a semisimple Jordan algebra, and all (semisimple) simple algebras are either Jordan algebras, flexible algebras of degree two, or simple quasi-associa tive algebras (that is, algebras obtained from simple associative algebras by redefining the product to be $x \cdot y=\lambda x y+(1-\lambda) y x$ for $\lambda$ in the base field). This reduces the study of power associative algebras defined by sets of identities to the study of the radical and the proof of trace-admissibility. It shows that algebras defined by new sets of identities will either require study by a new tool or will yield no new simple algebras. (Received April 7, 1949.)

\section{7t. P. T. Bateman and Sarvadaman Chowla: Averages of char-} acter sums.

The authors consider the arithmetic means of the sums $S(n)=\sum_{m=1}^{n} \chi(m)$, where $\chi$ is a primitive residue character modulo $k$. Among the results obtained are a stronger form of Paley's $\Omega$ result for $S(n)$ and, for the case $\chi(-1)=1$, a simple proof of Davenport's theorem that $|L(s, \chi)|\left\langle C k^{(1-\sigma) / 2}\right.$, where $s$ is a fixed complex number such that $0<\sigma=R(s)<1$ and $C$ depends only on $s$. (Received March 18,1949.)

328t. P. T. Bateman and Sarvadaman Chowla: Remarks on the size of $L(1, \chi)$.

Among other things it is proved that $\left|\sum_{n=1}^{\infty}(n / p) / n\right|<C \log p /(\log \log p)$ for infinitely many primes $p,(n / p)$ being the Legendre symbol. (Much better results in this direction are of course known for real primitive characters to unrestricted moduli.) In the other direction it is proved that if $\chi$ is a primitive residue character modulo $k$, then $L(1, \chi)<C \phi(k) \log k / k$, where $L(s, x)$ is Dirichlet's function, $\phi$ is Euler's function, and $C$ is a constant; this is valuable for those $k$ which have many distinct prime factors. (Received March 18, 1949.)

329t. P. T. Bateman and Sarvadaman Chowla: Proof of a statement of Paley.

The statement proved is that if $\chi$ is a primitive residue character modulo $k, b$ is a fixed positive number, and $n<k^{1 / 2}(\log k)^{b}$, then $\left|\sum_{m-1}^{n} \chi(m)\right|<C k^{1 / 2} \log \log k$, where $C$ is a constant depending upon $b$. (Received March 18, 1949.)

330t. Richard Bellman: Ramanujan functions and expansions in algebraic number fields. Preliminary report.

It is shown that Ramanujan functions, $C_{q}(n)$ (cf. Hardy, Ramanujan's work, 
\$11), can be defined over any algebraic number field, and that expansions analogous to those found by Ramanujan for $\sigma_{-s(n)}, d(n)$ and other number-theoretic functions hold for the arithmetic functions $s_{-8}(a)=\sum N(b)^{-s}, D(a)=\sum 1,\left(D(a)=S_{0}(a)\right)$, where the summation is over the ideals $b$ that divide the ideal $a$. These expansions can then be used to obtain mean values (cf. Bellman, Proc. Nat. Acad. Sci. U.S.A. (1948)). (Received March 21, 1949.)

\section{1t. R. C. Buck: Canonical mappings and algebraic structures. I.}

Let $S$ and $K$ be sets, $S \subset K$, such that no element of $S$ is a subset of $K$. The hierarchy of $S$ is defined to be the minimal set $h S$ such that: (1) $S \subset h S \subset K$, (2) if $A \subset h S$ and $P(A) \subset K$, then $P(A) \subset h S$. Denote by $A: B$ the set of all functions mapping $A$ into $B$. Then to each $f \in A: B$ there corresponds a unique function $\bar{f} \in h A: h B$, the maximal canonical extension of $f$. An element $\alpha$ of $h S$ is said to be canonical if $\bar{f}(\alpha)=\alpha$ for every $f$ which is a one-to-one mapping of $S$ onto $S$. It is then shown that a large body of familiar structures, some of which are termed "natural" in the EilenbergMacLane theory, are, in the present sense, canonical elements, mappings, or equivalences. Illustration: Let $G$ and $F$ be sets, $G^{*}=G: F, G^{* *}=G^{*}: F$. Then any multiplication $\bigcirc$ on $G$ induces canonically a corresponding operation $\otimes$ on $G^{* *}$ which agrees with $\bigcirc$ under the canonical map of $G$ into $G^{* *}$. In the familiar case, $\otimes$ is convolution. (Received March 18, 1949.)

\section{2t. R. C. Buck: Canonical mappings and algebraic structures. II.}

An algebraic structure on the set $S$ is a subset $\mathcal{S}$ of $h S$, the hierarchy of $S$. Two algebraic systems $(A, \mathcal{A})$ and $(B, B)$ are isomorphic if there is a function $f$ which maps $A$ one-to-one onto $B$ such that $\bar{f}$ maps $A$ onto $B$. Let $R=E: S$, the cartesian product of $S$ over $E$. Problem: given a structure on $S$, is it possible to describe a general procedure for canonically inducing a "corresponding" structure on $R$, and which sorts of structure are admissible? Partial answers are given for this decision problem in the case of "elementary" algebras and topological algebras, formulated in terms of an analysis of the structure by canonical elements in general relation algebras. Example: if $(S, \mathcal{S})$ is an elementary algebra, then any statement about the structure of $S$ which may be expressed as a conjunction of statements $p \rightarrow q$, where $p$ and $q$ are conjunctions of atomic statements, is admissible. (Received March 18, 1949.)

333t. Leonard Carlitz: Congruences for the coefficients of the Jacobi elliptic functions.

Let $k^{2}$ be integral $(\bmod p)$, where $p$ is a fixed odd prime, and put $\mathrm{sn}^{h}\left(x, k^{2}\right)$ $=\sum_{m} a_{m}^{(h)} x^{m} / m !$, so that the $a_{m}^{(k)}$ are integral (mod $\left.p\right)$. It is proved that $\sum_{i=0}^{r}(-1)^{i} C_{r, i} a_{p}^{r-i} a_{m+i(p-1)}^{(h)} \equiv 0\left(\bmod p^{r}\right)$ for $m \geqq r \geqq 1$. Using this result and the multiplication theorem for $\mathrm{sn} x$, it is proved that $\sum_{i=0}^{r}(-1)^{i} C_{r, i} a_{p}^{r-i} \tau_{m+i(p-1)} \equiv 0$ $\left(\bmod p^{r}\right)$, where $m>r \geqq 1, p-1 \nmid m, \tau_{m}=\beta_{m} / m$ and $x / \operatorname{sn} x=\sum \beta_{m} / x^{m} / m !$. (Received April 8, 1949.)

\section{Mary P. Dolciani: On Waring's problem with non-integral ex- ponents.}

Let $c$ be a real, non-integral number greater than one. B. Segal (Ann. of Math. vol. 36 (1935) pp. 507-520) has discussed the number of representations of any suffciently large positive integer in the form $\sum_{i=1}^{m}\left[x_{i}\right]$ where each $x_{i}$ is an integer. The result which he obtained corresponds fairly well to the inequality $G(k) \leqq(k-2) 2^{k-1}+5$ by Hardy and Littlewood in Waring's problem with integral $k$ th powers. The author 
improves Segal's result by employing methods and inequalities developed largely by Vinogradow. (Received March 18, 1949.)

335t. Herbert Federer and Bjarni Jónsson: Some properties of free groups.

In this paper the methods introduced into the theory of free groups by Jacob Nielsen are applied and extended. It is shown that if Nielsen reductions are applied at random to a finite sequence of elements of a free group, then the process will terminate after finitely many steps and yield a free generating set for the subgroup generated by the given elements. It is proved that if $G$ is freely generated by the set $X$ and if a subgroup $H$ of $G$ is well ordered in any manner consistent with $X$-length, then $H$ is freely generated by the set of all those elements which are not expressible in terms of preceding elements. The homomorphisms of free groups into free groups are completely described by the theorem that if $f$ is a homomorphism of a free group $G$ onto a free group $H$, then $G$ is expressible as a free product of two subgroups such that $f$ maps one factor isomorphically onto $H$ and $f$ maps the other factor onto the identity element of $H$. The answer to the question whether a given finite subset of a free group generates a free factor of this group is shown to be finitely computable. (Received February 15, 1949.)

\section{David Gale, H. W. Kuhn, A. W. Tucker: Symmetrizing a game.}

Let $G$ be a zero-sum two-person game with $m$ pure strategies for "white" and $n$ for black." Form a symmetric game $S$ with $m+n+1$ pure strategies for each player to correspond, respectively, to the $m$ white and the $n$ black strategies of $G$ and the privilege of "calling off." For a white strategy against a black, or vice versa, the pay-off in $S$ is just that in $G$; against a white or a black strategy a call-off wins or loses one unit, respectively; otherwise the pay-off in $S$ is zero. Theorem: A necessary and sufficient condition that the game $G$ have a positive value $v$ for white and have optimal mixed strategies $\left(x_{1}, \cdots, x_{m}\right)$ and $\left(y_{1}, \cdots, y_{n}\right)$ for white and black, respectively, is that the symmetric game $S$ have an optimal mixed strategy proportional to $\left(x_{1}, \cdots, x_{m}, y_{1}, \cdots, y_{n}, v\right)$. (Received March 18, 1949.)

\section{7t. W. J. LeVegue: On number-theoretic functions of two vari- ables.}

The author investigates the possibility of extending the definition of multiplicativity for functions of one variable to functions of two variables, under the restriction that the function corresponding to $\sum_{d \mid a} f(d)$ should also then be multiplicative in the same sense. It is shown that of the four definitions proposed, the only one satisfying this requirement is such that every function $f$ of this sort is the product of two multiplicative functions of one variable, that is, that $f(a, b)=g(a) h(b)$. It is also shown that if $f(a, b)$ is a function of the single variable $d=(a, b)$, say $f(a, b)=\psi(d)$, and if $\sum_{t=1}^{\infty} \psi(t) / t^{2}$ converges absolutely to the number $A$, then $\lim \left(1 / n^{2}\right) \sum_{a, b=1}^{\infty} \psi((a, b))$ $=6 A / \pi^{2}$. (Received March 15, 1949.)

\section{8t. W. J. LeVeque: On the number of representations of an integer as a sum of consecutive integers.}

Let $\gamma(m)$ be the number of representations of $m$ in the form $(r+1)+(r+2)+\cdots$ 
$+s$, where $s>r \geqq 0$. It is shown that $(1 / n) \sum_{1}^{*} \gamma(m)=(1 / 2) \log n+(2 \gamma+2 \log 2-1) / 4$ $+O\left(n^{-1 / 2}\right)$, and that if $s_{n}(\omega)$ is the number of positive integers $m \leqq n$ for which $\gamma(m) \leqq 2^{\log \log n+\omega(\log \log n)^{1 / 2-1}}$, then $\lim _{n \rightarrow \infty} s_{n}(\omega) / n=1 /(2 \pi)^{1 / 2} \int_{-\infty}^{\omega} e^{-x^{2} / 2} d x$, for every real $\omega$. The proofs depend on the relation $\gamma(m)=\tau(\bar{m})$, where $\tau(n)$ is the number of divisors of $n$, and $\bar{m}$ is the largest odd divisor of $m$. Generalizations are indicated for the number of representations of $m$ as a sum of integers in any of the arithmetic progressions with fixed difference $d>1$. (Received March 15, 1949.)

339. Pasquale Porcelli and Gordon Pall: $A$ theorem on quadratic residues.

By using a fundamental property of Farey Series the following theorem is proved: "If $p$ is an odd prime and $D$ is the quadratic residue of $p$, and if the integers $h$ and $k$ satisfy $h-1 \leqq p$ and $k=[p / h]$, then at least one of $1^{2}, 2^{2}, \cdots, k^{2}$ will be congruent to at least one of $D, 4 D, \cdots,(h-1)^{2} D$. The case $h=2$ is well known. (Received March 16, 1949.)

\section{R. D. Schafer: Inner derivations of non-associative algebras.}

A definition of inner derivation for non-associative algebras is given, and is applied to alternative and Jordan algebras. (Received April 21, 1949.)

\section{1t. G. Szekeres: Determination of a certain family of finite meta- belian groups.}

The object of the author is to determine all the finite abstract groups $G$ which have an abelian invariant subgroup $A$ with cyclic quotient group $F=G / A$. It is assumed that for any $\left.p \mid o(A), p^{2}\right\}_{o}(F)$. The algebraic equivalent of the problem is to set up all the finite abelian operator $p$-groups whose ring of operators is homomorphic to $R=I[x] /\left(x^{n}-1, p^{h}\right)$ where $I[x]$ is the ring of polynomials in $x$ with integral coeffcients, $p$ is a prime and $\left(n, p^{2}\right)<p^{2} . R$ is not a principal ideal ring, hence an indecomposable $R$-group is not necessarily "cyclic." The different types of indecomposable $R$-groups are completely determined and characterized by a set of numerical invariants. The solution for the corresponding abstract group-theoretical problem is then obtained in a fairly straightforward manner. Among the numerous applications perhaps the most significant is the determination of all the p-groups which have abelian subgroups of index $p$. An asymptotic formula is obtained for the number of such groups of order $p^{n}$ if $n$ is fixed and $p$ is large, also an estimate for the order of magnitude of this number if $p=2$ and $n$ is large. (Received February 11, 1949.)

\section{ANALysis}

\section{J. Aczel and P. C. Rosenbloom: On quasi-linear operations.}

Let $S$ be a linear vector space over the field of real numbers, $E$ be a convex subset of $S$, and let $U$ be a real-valued function on $E$ such that (1) $U\left(\left(f_{1}+g\right) / 2\right)=U\left(\left(f_{2}+g\right) / 2\right)$ whenever $f_{1}, f_{2}, g \in E$ and $U\left(f_{1}\right)=U\left(f_{2}\right),(2) U(t f+(1-t) g)$ is continuous in $0<t \leqq 1$ for every $f, g \in E$. Then there is a linear functional $L$ on $S$ and a strictly monotonic continuous function $\phi$ such that $U(f)=\phi(L(f))$ for all $f \in E$. This implies results of de Finetti and others on mean values. Conditions are obtained under which (1) and the monotonicity of $U$ in a suitable sense imply (2). A representation theorem is also obtained in the case of an operation $U$ whose range is an arbitrary linear topological space. (Received February 15, 1949.) 


\section{Lipman Bers: Boundary value problems for minimal surfaces with a singularity at infinity.}

The method announced previously for proving the existence of a gas flow obeying the linearized pressure-volume relation past a given convex obstacle (see the paper On existence theorem in two-dimensional gas dynamics to appear in the Proceedings of the First Symposium on Applied Mathematics) is extended to various other boundary value problems for minimal surfaces with a prescribed singularity at infinity. A typical problem is: to determine a minimal surface which winds itself around a given cylinder, cuts the cylinder under a right angle, possesses at infinity a tangent plane orthogonal to the directrix of the cylinder. Existence theorems (but no uniqueness theorems) are proved for this and other problems involving the integration of the minimal surface equation in a domain exterior to a curve $P$. The condition that $P$ be convex used in the previous paper is replaced by a weaker (though probably still unnecessarily restrictive) condition. The existence proof consists in reducing the boundary value problem to a mapping problem which is shown to be equivalent to a nonlinear integral equation. Using the Léray-Schauder method and the theory of conjugate functions, it is shown that the integral equation has a solution. (Received March 18, 1949.)

344t. Salomon Bochner and K. Chandrasekharan: On Fourier series of $L_{2}$ functions.

If $f(x)=f\left(x_{1}, \cdots, x_{k}\right)$ is a periodic function in each variable, belonging to the Lebesgue class $L_{2}$, and if $\left(^{*}\right) \int_{u}^{2 u}\left\{S^{\delta}(R)\right\}^{2} d R=o(u)$ as $u \rightarrow \infty$, for some $\delta>(k-1) / 2$, where $S^{\delta}(R)$ is the $\delta$ th Riesz mean of the Fourier series of $f$, when summed spherically, then $\int_{0}^{t}\left\{f_{p}(u)\right\}^{2} d u=o(t)$ for $p=\delta+(3-k) / 2, t \rightarrow 0$, where $f_{p}(u)$ is the $p$ th spherical mean of $f$; and the statement holds for $\delta=(k-1) / 2$ provided that $\left(^{*}\right)$ holds uniformly in an interval $(k-1) / 2 \leqq \delta<\delta_{0}$. This is reciprocal to a previous theorem of the authors (Ann. of Math. vol. 49 (1948) pp. 966-978), and seems to be new even in the case of one variable. (Received March 16, 1949.)

\section{W. M. Boothby: Topology of the level curves of harmonic func- tions with critical points.}

It was proved by W. Kaplan, Trans. Amer. Math. Soc. vol. 63 (1948) pp. 514-522, that (1) for every curve family $F$ filling the $x y$-plane which is regular (that is, locally homeomorphic to parallel lines) at every point of the plane, there exists a simplyconnected domain $D$ of the $x y$-plane and a homeomorphism carrying the plane onto $D$ such that $F$ is transformed onto the family of level curves of a function $f(x, y)$ harmonic in $D$; and (2) the family $F$ may be decomposed into non-overlapping, simplyconnected subfamilies in each of which $F$ is homeomorphic to the parallel lines of a half-plane. (1) gives a topological characterization of the level curves of a harmonic function with nonvanishing derivatives in a simply-connected domain; and (2) gives a decomposition of the Riemann surface of the inverse of a function which is analytic with nonvanishing derivative in a simply-connected domain into fundamental domains. In the present paper the author extends (1) and (2) to the case of curve families with a finite or infinite number of isolated singularities of the saddle or branch point type, and incidentally proves that a family of this type is homeomorphic to the solution family of a system of differential equations. (Received February 23, 1949.)

\footnotetext{
346t. D. G. Bourgin: Approximately multiplicative transformations on continuous function rings.
} 
Several definitions are given for approximately multiplicative transformations. For instance, the departure from strict multiplicativity of the transform of a product may be dominated by the infimum of one function multiplied by the norm of the other when, say, the underlying spaces are compact. Under weak auxiliary conditions the homeomorphism of the spaces can be asserted. (Received March 28, 1949.)

\section{7t. O. J. Farrell: On approximation by nonvanishing functions. I.}

Let $w=f(z)$ be analytic and bounded and have $k$ zeros in a limited simply connected region $G$. Let $S$ denote the closed set of functions $F(z)$ which are analytic in $G$ and vanish there either identically or not at all. For every $F(z)$ there is a $\mu=$ upper bound $[|F(z)-f(z)|, z$ in $G]$ and there is at least one $F(z)$ for which $\mu$ equals the greatest lower bound $M$ of all $\mu$. Let $R$ denote the Riemann configuration over the $w$-plane onto which $G$ is mapped by $w=f(z)$. Let $w=\delta$ be a point nearest the origin $w=0$ from among the boundary points of $R$ which do not correspond also to interior points of $G$. Let $D$ denote the largest of the radii of $k$-valence (see Seidel and Walsh, Trans. Amer. Math. Soc. vol. $52(1942)$ p. 162) at the points $w_{0}^{(1)}, w_{0}^{(2)}, \ldots, w_{0}^{(m)}$ of $R$ which lie over the origin $w=0$. It is found that $D \leqq M \leqq|\delta|$ and that every function $F_{1}(z)$ for which upper bound $\left[\left|F_{1}(z)-f(z)\right|, z\right.$ in $\left.G\right] \leqq D^{\prime}<D_{k}\left(w_{0}^{(h)}\right)$ has at least as many zeros in $G$ as the number of sheets belonging to the region $R_{k}\left(w_{0}^{(h)}\right)$. In this initial paper better appraisals on $M$ are obtained in certain restricted cases. Approximation by nonvanishing rational functions is considered. (Received March 17, 1949.)

\section{O. J. Farrell: On approximation to a k-valent function by func-} tions of lesser valence. I.

Let $w=f(z)$ be analytic and bounded and $k$-valent, $1<k$, in a limited simply connected region $G$. The class of functions which are analytic and either $h$-valent at most or identically constant in $G$ form a closed set $T_{h}$. For every function $g(z)$ of this set there is a $\lambda_{h}=$ upper bound $[|g(z)-f(z)|, z$ in $G]$ and in the set $T_{h}$ there is at least one function whose $\lambda_{h}$ equals the greatest lower bound $L_{h}$ of all $\lambda_{h}$. With each point $w=w_{0}$ covered by the Riemann configuration $R$ onto which $G$ is mapped by $w=f(z)$ there can be associated a greatest radius $C_{j}\left(w_{0}\right)$ of precise $j$-coverage. Let $C_{j}$ denote the greatest of all $C_{j}\left(w_{0}\right)$ and $C_{j}^{*}$ the greatest of all $C_{i}$ for which $j \leqq i$. Denote by $K$ the radius of the smallest circular biscuit cutter which will contain $R$. For $h<k$ it is found that $C_{h+1} \leqq L_{h} \leqq K$. Some results are also obtained on the order of multivalence of approximating functions. Included is an initial study of approximation by rational functions of specified valence. (Received March 17, 1949.)

\section{Gerald Freilich: On the measure of Cartesian product sets.}

Let $F_{n}^{k}$ represent the $k$-dimensional integral-geometric (Favard) measure over Euclidean $n$-space, $E_{n}$ (see H. Federer, The $(\phi, k)$ rectifiable subsets of $n$-space, Trans. Amer. Math. Soc. vol. 62 (1947) p. 122). If the following five conditions hold: (1) $A$ and $B$ are perpendicular subspaces of $E_{n}$ of dimensions $a$ and $b$ respectively, (2) $k \leqq a$, (3) $S$ is an $F_{n}^{k}$-measurable subset of $A$ with $F_{n}^{k}(S)<\infty$, (4) $T$ is an $F_{n}^{b}$-measurable subset of $B$ with $F_{n}^{b}(T)<\infty$, (5) $S+T=\{(s+t) \mid s \in S$ and $t \in T\}$, then it is shown that $F_{n}^{k+b}(S+T)=F_{n}^{n}(S) \cdot F_{n}^{b}(T)$. Besicovitch's example, which shows that the correspond ing statement for Hausdorff measure is false, is examined in detail. In addition, for $X \subset E_{n}, k \leqq m \leqq n$, a new formula is obtained which, in terms of the multiplicity integral with respect to $F_{m}^{k}$ of the perpendicular projections of $X$ into $m$-planes, expresses $F_{n}^{k}(X)$ as an integral over the set of all these $m$-planes. (Received March 1, 1949.) 
350t. W. H. Ingram: The 2-point 1-dimensional generalized boundary value problem.

The problem $d y-d H(x, \mu)=0, L y(a)+R y(b)=0, H$ an $n \times n$ matric function in $\mathfrak{B}^{\prime}(x) \subset \mathfrak{B}(x)$ uniformly and analytic in $\mu, d y=y(x+)-y(x)+y^{\prime}(x+) d x, d x>0$, $y^{\prime}(x+) \equiv \lim [y(x+\delta)-y(x+)] / \delta, \delta>0$, requires the integration of weighted differentials $f d g$ in which $f$ and $g$ have simultaneous discontinuities. The integral $\int_{a}^{b} f d g$ is the Smith-More limit of the interval function $\sum f^{U}\left(x_{i}+\right)\left[g\left(x_{i+1}\right)-g\left(x_{i}\right)\right] f^{v}(x+)$ $\equiv \operatorname{glb}_{\delta>0} \operatorname{lub}_{\xi \text { in } S, x<\xi \leqq x+\delta} f(\xi), g(x)$ is in $2 \mathbb{B}(x)$ the class of functions of bounded variation, and $f$ is any bounded function in $\mathfrak{D}_{(2)}$, the class consisting of all functions on $\subseteq$ to $\Re$ with the property that to each $\epsilon>0$ corresponds a set $\sigma_{\epsilon}$ of real points on $[a b]$ such that the oscillation, defined on $\subseteq$ to $\Re$, at every other real point is less than $\epsilon$. If $g$ is in $\mathfrak{B}^{\prime}$, the continuous part of $g$ has a finite derivative everywhere and the discontinuities of $g$ form a point-set of the first species. The set $\Phi$ contains the real numbers and all satellite numbers $x(+), x(-)$; if $x_{1}(+)$ denotes the point, asserted to exist, common to all the open intervals $\left(x_{1}<x<x_{1}+\delta\right), \delta>0$, the corresponding number is $x_{1}(+)$; similarly for $x_{1}(-)$. The Archimedean property does not extend to $\subseteq$ and $\mathfrak{S}$ is not a field, but $(\mathfrak{S},>)$ forms a linearly ordered set having axcribable requisite metric properties and the Dedekind property is asserted for $\mathfrak{S}$. (Received April 8, 1949.)

351t. Hermann Kober: On a monotone singular function and on the approximation of analytic functions by nearly analytic functions in the complex domain.

First certain monotone functions $\omega(t)$ are treated which are continuous and are constant in $\langle 0, T\rangle$ on the intervals of an open set of measure $T(0<T<\infty)$. A subclass of them is well known. Here they are dealt with from two new points of view. (A) The inverse functions, strictly increasing jump-functions, admit of plain representations. (B) $\omega(t)$ is defined by two very simple functional equations; it is subadditive, and it satisfies some more equations and inequalities. Then by means of $\omega(t)$, "nearly analytic" functions are constructed. They satisfy a Lipschitz condition, and this property and others are discussed. If $f(z)$ and $g(z)(z=x+i y)$ are any two functions which, for $|z|<1$, are analytic and have bounded derivatives, then, given $\epsilon>0$, there exists a nearly analytic $H(z)$ such that $|f(z)-H(z)|<_{\epsilon}(|z| \leqq 1)$ and that $H^{\prime}(z)=g^{\prime}(z)$ for almost all $z$. (Received February 17, 1949.)

352t. Norman Levinson. An ordinary differential equation with an interval of stability, a separation point, and an interval of unstable oscillation.

The second order differential equation $\epsilon u^{\prime \prime}-4 u^{\prime} /\left[3+\left(u^{\prime}\right)^{4}\right]+u=0$, where $u^{\prime}=d u / d x$, is considered for small $\epsilon>0$. A solution with initial values at $x=0$ given by $u_{0}$ and $u_{0}^{\prime}$ will, if $\left|u_{0}\right|<1$ and $\left|u_{0}^{\prime}\right|$ is not large, stay very close to a monotone solution $z(x)$ of the degenerate equation $-4 z^{\prime} /\left(3+\left(z^{\prime}\right)^{4}\right]+z=0$ obtained by putting $\epsilon=0$. However as $z(x) \rightarrow 1$ (or -1 ), which will occur for some finite value of $z=z_{1}, z(x)$ cannot be continued further in the range of real values. As $x$ gets near to $x_{1}$ the solution $u(x)$ nears 1 and then turns rapidly downward to approximately -1 . The solution $u(x)$ then continues to oscillate rapidly between approximately +1 and -1 with a "period" of approximately $2 \pi \epsilon^{1 / 2}$. In the range $0<x<x_{1}, u(x)$ is highly stable. Beyond $x_{1}, u(x)$ is highly oscillatory and is unstable in "phase." Separation between the stable and oscillatory intervals occur at a value of $x$ which tends to $x_{1}$ as $\epsilon \rightarrow 0$. The 
method can be used for a broad class of equations of the form $\epsilon u^{\prime \prime}+f\left(x, u, u^{\prime}, \epsilon\right)=0$. (Received March 4, 1949.)

353t. Norman Levinson: Boundary value problem for $\epsilon \Delta u+A(x, y) u_{x}$ $+B(x, y) u_{y}+C(x, y) u=D(x, y)$ for small $\epsilon$.

Under quite general conditions it is shown that the solution $u(x, y, \epsilon)$ of the first boundary value problem for small $\epsilon>0$ exists in a simply or multiply connected domain $R$ and that at interior points in most of $R, \lim u(x, y, \epsilon)$ as $\epsilon \rightarrow+0$ exists and is a solution of the first order equation with $\epsilon=0, A U_{x}+B U_{y}+C U=D$ which assumes the given boundary value on certain arcs of the boundary. Let $S$ be the boundary of $R$. More specifically $\left({ }^{*}\right) u(x, y, \epsilon)=U(x, y)+z(x, y, \epsilon)+O\left(\epsilon^{1 / 2}\right)$ for small $\epsilon$ uniformly in each of a finite number of regions $Q_{i}$ into which $R+S$ can be divided where $z$ is of the form $h(x, y) \exp (-g(x, y) / \epsilon)$ near certain arcs of the boundary and is zero on the rest of the boundary. Moreover $g=0$ on these arcs of the boundary and $g>0$ in $R$. The term $z$ is the "boundary layer" term. More precise results are possible where the error is $O\left(\epsilon^{n+1 / 2}\right)$ for any fixed $n$ and more general cases of the equation can be handled. In the exact formulation the solutions of the equation $d x / A=d y / B$ play a f undamental role. (Received March 4, 1949.)

\section{4t. Norman Levinson: The inverse Sturm-Liouville problem.}

It is assumed that the boundary conditions and characteristic values of $y^{\prime \prime}+(\lambda-P(x)) y=0,0 \leqq x \leqq \pi$, are known and the problem is to find $P(x) . P(x)$ is assumed integrable over $(0, \pi)$. Let the boundary conditions be $y(0) \cos \alpha+y^{\prime}(0)$ $\sin \alpha=0, y(\pi) \cos \beta+y^{\prime}(\pi) \sin \beta=0$. Then if $\left(^{*}\right) P(\pi-x)=P(x)$ and if $\alpha+\beta=\pi, P(x)$ is uniquely determined by the spectrum. It is also shown without assumption $\left(^{*}\right)$ that if the spectrum is known for the above boundary condition and for another boundary condition with $\beta$ replaced by $\gamma$ (where of course $\sin (\beta-\gamma) \neq 0$ ) then $P(x)$ is uniquely determined. These results are similar to earlier results of Borg. The method used is a simplified form of a proof that under certain conditions for the differential equation with $0<x<\infty$, the asymptotic phase determines $P(x)$ and makes use of a pseudo Green's function involving $\lambda$ where $\lambda$ is regarded as a complex variable. (Received March 4, 1949.)

355t. Norman Levinson: Uniqueness of potential for given asymptotic phase with inverse square term present in wave equation.

The solution $y(x, \lambda)$ of $y^{\prime \prime}+\left(\lambda^{2}-V(x)-l(l+1) / x^{2}\right) y=0$ with boundary condition $y(x, \lambda) / x^{l+1} \rightarrow 1$ as $x \rightarrow+0$ exists if $\int_{0}^{\infty} x|V(x)| d x<\infty$. Moreover for any $\lambda \rightarrow 0$, $\lambda^{l+1} y(x, \lambda)-A(\lambda) \sin (\lambda x-l \pi / 2-\phi(\lambda)) \rightarrow 0$ as $x \rightarrow \infty$. The function $\phi(\lambda)$ is the asymptotic phase. If $V(x)+l(l+1) / x^{2} \geqq 0$ then $\phi(\lambda)$ determines $V(x)$ uniquely. Also there exists a function of $\lambda, F(\lambda)$, analytic in the upper half $\lambda$ plane and free of zeros such that on the real axis, $F(\lambda)=A(\lambda) \exp (i \phi(\lambda))$. Thus $\phi(\lambda)$ and $\log A(\lambda)$ are conjugate functions on the real axis. The proof is similar to that for the case $l=0$ in a paper to appear shortly in Det. Kgl. Danske Videnskabernes Selskab. (Received March 4, 1949.)

\section{E. R. Lorch: Differentiable inequalities. Preliminary report.}

The type of inequality treated is $\sum_{1}^{n} x_{i} y_{i} \leqq \psi(x) \phi(y)$. Here $x$ represents the vector $\left(x_{1}, \cdots, x_{n}\right)$ and $\phi$ and $\psi$ are functions which characterize the inequality. Contrary to first impressions, existing means do not seem to provide "correct" methods for 
establishing these inequalities. The method used applies to convexity and concavity. The principal features are the use of differentiability, homogeneity, the definition of convexity (concavity) by means of positive definite quadratic forms, and the introduction of a characteristic transformation between subsets of two spaces. Consider the case of convexity. Let $G(x)=G\left(x_{1}, \cdots, x_{n}\right)$ be a function positively homogeneous of degree $r>1$ and differentiable sufficiently of ten; let $G_{i}(x)$ and $G_{i j}(x)$ denote $\partial G / \partial x_{i}$ and $\partial^{2} G / \partial x_{i} \partial x_{j}$. Let $\Omega$ be a ray-space in the $x$ space. Let $\sum G_{i j}(x) z_{i} z_{j}$ be a positive definite form for all $x \in \Omega$. Then $x_{i}^{*}=G_{i}(x)$ defines a 1-1 transformation of $\Omega$ to a rayspace $\Omega^{*}$ in the $x^{*}$ space. The inverse transformation is $x_{i}=F_{i}\left(x^{*}\right)$. The functional equation for $\phi(x)>0$ : $\max _{y}\left(\sum x_{i}^{*} y_{i}\right) / \phi(y)=\left(\sum x_{i}^{*} x_{i}\right) / \phi(x)$ has a unique solution $\phi(x)=\left[\sum x_{i}^{*} x_{i}\right]^{1 / r}$. Setting $\psi\left(x^{*}\right)=\left[\sum x_{i}^{*} x_{i}\right]^{(r-1) / r}$ we have $\sum x_{i}^{*} y_{i} \leqq \psi\left(x^{*}\right) \phi(y)$ for all $y \in \Omega, x^{*} \in \Omega^{*}$. The situation between the $x$ and $x^{*}$ space is completely symmetric. The theory of approximation of non-differentiable by differentiable inequalities will be treated in another communication. (Received March 21, 1949.)

357. G. W. Mackey: On the unitary representations of subgroups of locally compact groups. Preliminary report.

Let $G$ be a separable locally compact group and let $G_{0}$ be a closed subgroup of $G$. Let $h \rightarrow V_{h}$ be a suitably continuous representation of the ring of all complex Borel functions on the space $G / G_{0}$ of right cosets of $G_{0}$ into the ring of all bounded linear operators on a Hilbert space $H$. Let $\sigma \rightarrow U_{\sigma}$ be a continuous unitary representation of $G$ in $H . V, U$ is called a system for $G, G_{0}$ if $U_{\sigma} V_{h}=V_{h \sigma} U_{\sigma}$ for all $h$ and $\sigma$ where $h_{\sigma}(x)=h(x \sigma)$. Every system has associated with it in a natural way a continuous unitary representation of $G_{0}$ in a Hilbert space $H^{\prime}$. Two systems are unitary equivalent if and only if the corresponding representations of $G_{0}$ are unitary equivalent. A system is irreducible if and only if the corresponding representation of $G_{0}$ is irreducible. Under a (probably superfluous) hypothesis regarding the possibility of selecting an element from each coset of $G_{0}$ in a suitably regular manner it can be shown that every continuous unitary representation of $G_{0}$ is associated with some system. If $G_{0}$ is the identity these results are equivalent to a theorem announced in an earlier abstract (Bull. Amer. Math. Soc. Abstract 55-3-153). (Received March 21, 1949.)

358t. E. F. Moore: The two-dimensional measures of Carathéodory and Hausdorff. Preliminary report.

Let $C$ and $H$ be the two-dimensional measures of Carathéodory and Hausdorff over Euclidean 3-space $E_{3}$. It is shown that $C(A) \leqq H(A) \leqq\left(\pi\left(1+3^{1 / 2}\right) / 2\right) C(A)$ for all subsets $A$ of $E_{3}$, and that $0<C(A)<H(A)<\infty$ in case $A$ is the set of all those points of $E_{3}$ each of whose three coordinates is between 0 and 1 and has an octal expansion in which only the digits $0,1,2,3$ occur. Generalizations to higher dimensions are also considered. (Received February 21, 1949.)

359t. J. C. Oxtoby: A minimal set that is not metrically transitive.

In the compact metric space of all mappings $x(n)$ of the integers into the set $\{0,1\}$, acted on by the homeomorphism $T x=x(n+1)$, it is shown that there exists a (regularly) almost periodic point $x$ with the property that the sequence $x(n), n>0$, does not have a density. The orbit closure $X$ of any such point provides an example of a minimal set in which two distinct invariant Borel measures with $\mu(X)=1$ can be introduced. For the method used in defining these measures, see Oxtoby and Ulam (Ann. of Math. vol.40 (1939) pp. 560-566). The sum of these two measures is a measure with respect to which $T$ is not metrically transitive. (Received March 23, 1949.) 
360t. A. M. Peiser: Uniform approximations to a class of Bessel functions.

The functions $K_{n}(x, y)=(y / x)_{n}{ }^{2} I_{n}\left(2(x y)^{1 / 2}\right) \exp (-x-y), n=0,1,2, \cdots$, arise in the solution of the differential equation $\partial^{2} t / \partial x \partial y+\partial t / \partial x+\partial t / \partial y=0$. When boundary values $t(x, 0)$ and $r(0, y)$ are specified, $t(x, y)$ can be expressed in terms of integrals of the form $\int_{0}^{x} f_{n}(x-u) K_{n}(u, y) d u, n=0,1$, where $f_{n}$ is known. Boundary conditions of the form $t(x, 0)=a+b t(S-x, P)$ lead to the integral equation $F(x)$ $+b t(x, 0) e^{-P}+b \int_{0}^{x} t(u, 0) K_{1}(x-u, P) d u=t(S-x, 0)$, where $F$ is known. In either case, it is convenient to have approximations to $K_{n}$ which hold uniformly in $x$. In this paper, it is shown that $K_{n}(x, y)$ can be approximated uniformly in $0 \leqq x<\infty$, for sufficiently large values of $y$, by means of the normal probability function $\Phi(x)=\exp \left(-x^{2} / 2\right) /(2 \pi)^{1 / 2}$. It is shown, for example, that for $y \geqq 10, \mid K_{1}(x, y)$ $-\Phi\left((x-y) /(2 y)^{1 / 2}\right) /(2 y)^{1 / 2} \mid<0.167 y^{-1}+0.800 y^{-3 / 2}+0.694 y^{-1 / 2} e^{-\nu}$. A similar estimate is obtained for $K_{0}(z, y)$. Better approximations to $K_{n}$ are obtained by asymptotic series involving the successive derivatives of $\Phi(x)$. (Received February 23, 1949.)

361. F. W. Perkins: Mean value properties of polyharmonic functions. Preliminary report.

By the use of Almansi's theorem expressing a polyharmonic function in terms of harmonic functions, various mean value properties of harmonic functions are generalized for polyharmonic functions. Parts of the paper are related to earlier work of the author (Amer. J. Math. vol. 61 (1939) pp. 217-230) and to two papers by Poritsky (Amer. J. Math. vol. 54 (1932) pp. 667-691, and Trans. Amer. Math. Soc. vol. 43 (1938) pp. 199-225). (Received March 18, 1949.)

362t. Hans Rademacher and I. J. Schoenberg: Helly's theorems on convex domains and Tchebycheff's approximation problem.

The main purpose of the authors is to demonstrate the close connection between Helly's theorems on convex domains having a common point and the problem of best approximation in the sense of Tchebycheff. This new connection benefits both subjects thus brought into contact: One result is a direct geometric proof of Helly's theorems, which, unlike other proofs, makes no use of mathematical induction; another result is an application of Helly's theorems to derive properties of the polynomial of best approximation to discontinuous functions. As further examples of the versatility of Helly's ideas, simple proofs are given for a recent theorem of $M$. Dresher and T. E. Harris as well as for an interesting separation theorem of Paul Kirchberger (Dissertation, Göttingen, 1902). (Received March 16, 1949.)

\section{3t. J. F. Ritt: Abel's theorem and a generalization of one-parameter groups.}

For any positive integer $p$, an associative operation is defined which replaces any set of $p-1$ complex numbers by a set of $p$ numbers. The operation is found to be described by relations analogous to those satisfied by the integrals of the first kind on a Riemann surface of genus $p$. (Received March 24, 1949.)

\section{Arthur Sard: Remainders: functions of several variables.}

Remainders in the theory of approximation are of ten functionals which are linear on certain function spaces and zero whenever their argument is a polynomial of de- 
gree $n-1$. For spaces of functions of one variable, known mass and kernel theorems, due to F. Riesz, Peano, and Rémès, give integral forms of such functionals in terms of $n$th derivatives. The author extends the mass and kernel theorems to spaces of functions of several variables. Among the results is the following. Let $a, b, p, q$ be fixed: $0 \leqq a, b \leqq 1 ; p, q$ non-negative integers. Put $n=p+q$. Denote by $B_{p, q}$ the space of functions $x=x(s, t)$ for which the $n$th partial derivatives: $x_{n-j, j}(s, b), j<q ; x_{p, q}(s, t)$; $x_{i, n-i}(s, t), i<p$ exist and are continuous in $s,(s, t), t$, respectively, on $0 \leqq s, t \leqq 1$. (The order of differentiation in $x_{i, j}$ is restricted: If $i>p$ the last $i-p$ differentiations are to be with respect to $s$; likewise if $j>q$.) Define the norm in $B_{p, q}$ as $\|x\|=\max$ $\left[\left|x_{i, j}(s, t)\right|,\left|x_{i^{\prime}, j^{\prime}}(s, b)\right|,\left[x_{i^{\prime \prime}, j^{\prime \prime}}(a, t) \mid\right]\right.$, where the maximum is taken over $0<s$, $t \leqq 1, i \leqq p, j \leqq q, i^{\prime}+j^{\prime} \leqq n, i^{\prime}>p, i^{\prime \prime}+j^{\prime \prime} \leqq n, j^{\prime \prime}>q$. Let $R x$ be a functional, additive and continuous on $B_{p-1, q-1}, p \geqq 1, q \geqq 1$, and zero whenever $x=x(s, t)$ is a polynomial of degree $n-1$ in $(s, t)$. Then there exist kernels $f^{i}(s), j<q ; g(s, t) ; h^{i}(t), i<p$; such that $R x=\sum_{j<q} \int_{0}^{1} x_{n-j, j}(s, b) f^{j}(s) d s+\int_{0}^{1} \int_{0}^{1} x_{p, q}(s, t) g(s, t) d s d t+\sum_{i<p} \int_{0}^{1} x_{i, n-i}(a, t) h^{i}(t) d t$ whenever $z \in B_{\boldsymbol{p}, \boldsymbol{q}}$. The kernels are functions of bounded variation and are given by explicit formulas. (Received March 16, 1949.)

365. I. J. Schoenberg and Anne M. Whitney: On the positivity of translation determinants for Polya frequency functions.

A function $\Lambda(x),-\infty<x<\infty$, is called a Poblya frequency function (P.f.f.) if it is measurable, with $\int_{-\infty}^{\infty} \Lambda(x) d x=1$, and satisfies the condition: If $x_{1}<x_{2}<\cdots<x_{n}$, $y_{1}<y_{2}<\cdots<y_{n}$, the $D=\operatorname{det}\left\|\Lambda\left(x_{i}-y_{k}\right)\right\| \geqq 0, n=1,2, \cdots$. It was shown (Proc. Nat. Acad. Sci. U. S. A. vol. 33 (1947) pp. 11-17) that every P.f.f. $\Lambda(x)$ has a Laplace transform $\int_{-\infty}^{\infty} \exp (-x s) \Lambda(x) d x=1 / F(s)$, where $F(s)=\exp \left(-\gamma s^{2}+\delta s\right) \cdot \prod_{\nu_{-1}}^{\infty}\left(1+\delta_{\nu} s\right)$ $\exp \left(-\delta_{\nu} s\right)$ with all constants real, $\gamma \geqq 0,0<\gamma+\sum \delta_{\nu}^{2}<\infty$. The authors solve the problem of deciding when $D>0$. The results are as follows: 1 . If $\gamma>0$, then always $D>0$. 2. If $\gamma=0$ there are two cases: 2a. If $\sum\left|\delta_{\nu}\right|$ diverges then again always $D>0$. 2b. If $\sum\left|\delta_{\nu}\right|$ converges, then by a shift of origin assume that $\int_{-\infty}^{\infty} \exp (-x s) \Lambda(x) d x$ $=1 / \sum\left(1+\delta_{\nu} s\right)$. Let there be $k$ positive $\delta_{\nu}$ and $h$ negative $\delta_{\nu}(0 \leqq k, h \leqq \infty)$; then $D>0$ if and only if the $n$ inequalities (1) $x_{j-k}<y_{j}<x_{j+h}(j=1, \cdots, n)$ hold, where $x_{r}=-\infty$ if $r \leqq 0$ and $x_{r}=+\infty$ if $r>n$. The trivial case $k+h=1$, when $\Lambda(x)$ becomes a truncated exponential, is exceptional in that equality is allowed on one side of (1) depending on how $\Lambda(x)$ is defined at its discontinuity. These results contain as special cases all known positive determinants and many new ones. (Received March 21, 1949.)

366. Daniel Shanks: Mathematical sequences treated as transients. Preliminary report.

Given a sequence $A_{n}$, pass a curve through $2 K+1$ points $\left(n, A_{n}\right)$ of the sequence, $N-K \leqq n \leqq N+K$, of the form $A_{n}=B_{K N}+\sum_{i=1}^{K} a_{i} e^{\alpha_{i n}}$ with the $2 K+1$ unknowns $B_{K N}, a_{i}$ and $\alpha_{i}$, and solve for $B_{K N} . B_{K N}$ is the ratio of two $K+1$ order determinants whose elements are the $A$ 's and their differences. Two types of nonlinear sequence-tosequence transforms are $A_{N} \rightarrow B_{N N}$ and $A_{N} \rightarrow B_{K N}$ (with $K$ fixed). Both types may be iterated. The author shows the first-order iterated transform, $A_{N} \rightarrow B_{1 N} \rightarrow C_{1 N} \rightarrow \cdots$, to be a useful summation process. Applied to the first nine terms of the series $\sum_{\int_{0}^{\infty}}^{\infty}(-1)^{n}(n+1)^{-1}, \sum_{0}^{\infty}(-1)^{n} n !, 1 / 2+\sum_{0}^{\infty} B_{2 n}(2 n)^{-1}$ the transform gives $\ln 2$, $\int_{0}^{\infty} e^{-t}(1+t)^{-1} d t$, and Euler's $C$, correct to 9,6 , and 7 places, respectively. The transform $A_{N} \rightarrow B_{N N}$, when applied to the power series of analytic functions, yields known rational approximations to, and continued fraction representations for, these functions. Applications to other types of sequences, to iterative techniques, and to 
differential equations will be published later. (Received March 17, 1949.)

\section{C. F. Stephens: Nonlinear difference equations containing a parameter.}

This paper contains a quick and simple method of existence proof for solutions of the special class of nonlinear difference systems: $y_{i}(x+1)=x^{-1} \sum_{j=1}^{n} b_{i j}(x) y_{j}(x)$ $+x^{-1} A_{i 1}(x) p+f_{i}\left(y_{1}(x), \cdots, y_{n}(x) ; p ; x\right)$, where $f_{i}(0, \cdots, 0 ; 0 ; x) \equiv 0$. The functions $b_{i j}(x)$ and $A_{i 1}(x)$, where $A_{i 1}(x)$ can conceivably be identically zero, are any bounded and continuous functions of $x$; the $f_{i}$ are bounded, contain terms in the constant parameter $p$ alone, are continuous in $x$, begin with terms of the second degree in $y_{i}(x)$ and $p$, can be developed into convergent power series in the $y_{i}(x)$, and $p$ is the domain: $\left|y_{i}(x)\right| \leqq r_{i},|p| \leqq \rho,|x| \geqq K$. The proof that the given system has a solution which is continuous in $x$ and analytic in $p$ is based on a lemma which is similar to a result obtained previously by the author (Trans. Amer. Math. Soc. vol. 64 (1948) pp. 277278) and can be proved by similar methods. The transformation $y_{i}(x)=p z_{i}(x)$ is used to transform the above system into a system such that the result of the lemma is easily applied. (Received February 15, 1949.)

\section{8t. Walter Strodt: On a class of nonlinear difference equations in the complex domain.}

For any positive $\beta \leqq \pi / 2$ the function $g(x)$ is called $\beta$-admissible if for each positive $\gamma$ less than $\beta$ there is a $\xi \geqq 0$ such that $g(x)$ is analytic and bounded in the sector $|\arg (x-\xi)|<\gamma$, and if $g(x)$ approaches a limit as $x$ becomes infinite through positive values. Let $\Lambda\left(x, y_{1}, \cdots, y_{n}\right)$ be a polynomial in $y_{i}$ with $\beta$-admissible coefficients. Let $\omega_{1}=0$, and $\omega_{2}, \cdots, \omega_{n}$ be complex numbers with $\left|\arg \omega_{j}\right|<\beta$. Let $\mathcal{T}$ be the set of all $\beta$-admissible solutions of the difference equation (1) $\Delta\left[x, y\left(x+\omega_{1}\right), \cdots, y\left(x+\omega_{n}\right)\right]$ $=0$. If $\Lambda(+\infty, \sigma, \cdots, \sigma)$ is not identically zero, if its zeros $\sigma_{1}, \cdots, \sigma_{c}$ are all simple, and if $\sigma \Lambda\left(+\infty, \sigma_{k}, \cdots, \sigma_{k}\right) / \partial y_{1}$ is nonzero for every $k$, then $\mathcal{G}=\mathcal{G}_{1}+\cdots+\mathcal{G}_{c}$, where $\mathcal{T}_{k}$ is the set of all $\beta$-admissible solutions $y(x)$ of (1) such that $y(+\infty)=\sigma_{k}$. $\mathcal{T}_{k}$ is a nonempty finite-parameter family of functions, the number $q_{k}$ of complex parameters being the number of zeros of the exponential polynomial $\sum_{j=1}^{n}\left[\partial \Lambda\left(+\infty, \sigma_{k}, \cdots, \sigma_{k}\right) / \sigma y_{j}\right] \exp \left(\omega_{j} z\right)$ lying in the complement of the set $|\arg z|<\beta+\pi / 2$. (In terms of a suitable metric $\mathcal{V}_{k}$ is a topological manifold of $2 q_{k}$ dimensions.) This is proved by successive approximations using solutions of linear difference equations with constant coefficients. (Received February 9, 1949.)

369t. D. B. Sumner: An integral inversion formula for certain convolution transforms. Preliminary report.

The nucleus of the convolution transform $f(x)=\int_{-\infty}^{\infty} g(x-t) \phi(t) d t$ is defined in terms of the entire function $E(s)=s \cdot L(s) \cdot M(s)$ by $1 / E(s)=\int_{-\infty}^{\infty} \exp (-s t) g(t) d t$, where $L(s)=\prod_{1}^{\infty}\left[1-s / \lambda_{n}\right] e^{s / \lambda_{n}}, M(s)=\prod_{1}^{\infty}\left(1+s / \mu_{n}\right) e^{-s / \mu_{n}},\left\{\lambda_{n}\right\}_{1}^{\infty},\left\{\mu_{n}\right\}_{1}^{\infty}$ are increasing sequences of positive numbers, and $\sum \lambda_{n}^{-2}, \sum \mu_{n}^{-2}<\infty$. Under these assumptions Widder has shown that $E(D) \cdot f(x)$, interpreted as $\lim _{n \rightarrow \infty} E_{n}(D) \cdot f(x)$, inverts the transform. The canonical product $L(s) \cdot M(s)$ has finite order less than 2 , so that the function exp $\left(-k x^{2}\right) C(x) \in L(-\infty, \infty)$ for some positive $k$, and $C(x)$ $=\lim _{\alpha \rightarrow 0}(2 \pi)^{-1} \int_{-\infty}^{\infty} C(t) d t \int_{-\infty}^{\infty} \exp \left[-\alpha^{2} u^{2}+i u(x-t)\right] d u$. The operation $E(D) \cdot f(x)$ $=C(D) \cdot f^{\prime}(x)$ is interpreted as $\lim _{\alpha \rightarrow 0}(2 \pi)^{-1} \int_{-\infty}^{\infty} C(t) d t \int_{-\infty}^{\infty} \exp \left[-\alpha^{2} u^{2}-i u t\right] f^{\prime}(x+i u) d u$, and it is shown that this formula inverts the convolution transform. (Received March 21, 1949.). 


\section{Applied Mathematics}

\section{0t. Milton Abramowitz: Laguerre functions expressed in terms of Bessel-Clifford functions.}

Expansions are derived from the Laguerre functions $L_{\nu}^{(\mu)}(x)$ in terms of the Bessel-Clifford functions $F_{n}(\nu x)=(\nu x)^{-n / 2} J_{n}(\nu x)$. These expansions are valid for large values of $\nu$. It is also shown that the Hermite polynomials $H_{n}(x)$ of large order may be expressed in terms of spherical Bessel functions. (Received March 16, 1949.)

\section{1t. Carl Cohen: Integration of the generalized dilogarithm.}

The generalized dilogarithm $\mathrm{dl}^{(k)} x=\int_{0}^{z} \mathrm{dl}^{(k-1)} t \cdot t^{-1} d t, \mathrm{dl}^{(0)} x=-\log (1-x)$, plays a role in the theory of iterated integrals of expressions $R(x, \log (x-a)) ; R=$ rational. For $m>0, \int_{0}^{x} \mathrm{dl}^{(k)} t \cdot(t-c)^{m} d t, c$ a constant, may be expressed elementarily by $x, \log x$ and $\mathrm{dl}^{(i)} x, 0 \leqq i \leqq k$. Integrals involving the first dilogarithm only and $(t-c)^{-m}, m \neq 1$, can also be expressed elementarily by $\mathrm{dl}^{(1)} x, \log x$, and $x$. In this paper, it is shown that while by a classical result $\int_{0}^{x} \mathrm{dl}^{(1)} t \cdot(t-c)^{-1} d t$ involves a rational function of the second dilogarithm, it is impossible for $i \geqq 2$ to integrate $R\left(\mathrm{dl}^{(i)}(x-b)(x-a)^{-1}\right)$ in terms of higher dilogarithms and elementary functions, except the cases $a=b, a=0$, though it is possible to express $\int \mathrm{dl}^{(2)}(1-a x) \cdot x^{-1} d x$ and $\int \mathrm{dl}^{(2)} x \cdot(1-a x)^{-2} d x$ as $R\left(\mathrm{dl}^{(2)} x, \mathrm{dl}^{(1)} x, \log (1-x), x\right)$. (Received March 15, 1949.)

\section{A. H. Copeland: A cybernetic model of memory and recognition.}

The purpose of the author is to produce a consistency proof for certain assumptions concerning neural networks and to offer direction to future experimentation concerning the nervous system. To accomplish this a linear graph (neural network) is constructed which is capable of storing information, recognizing similarities between new and old experiences, reminiscing, and reasoning. The graph's memory is by association and it is shown that this association gives rise to a Boolean algebra which forms the basis of the reasoning mechanism. The Boolean algebra of reasoning is only indirectly related to the binary number system although this system plays an important role in the construction of the graph. In this model, inhibition is not produced by special inhibitory fibres or special inhibitory impulses but rather by differences in firing thresholds and by timing. The assumption concerning differential thresholds has some experimental justification and its consistency is established by the model. Incidentally with a moderate alteration the graph becomes the wiring diagram of an ultra rapid electronic device to do the work of an I. B. M. sorting machine. (Received March 18, 1949.)

373. A. E. Heins: $A$ boundary value problem in the theory of elastic stiffeners.

This problem at hand concerns the solution for the semi-infinite plane $-\infty<x$ $<\infty$ of the two-dimensional Laplace equation $\phi_{x x}+\phi_{y y}=0$ in which $y \geqq 0$ and $\phi(x, y)$ is subject to the following boundary conditions: (i) $\phi(x, y)=0, x<0, y=0$; (ii) $\phi(x, y)-\alpha \phi_{y}(x, y)=\beta, x>0, y=0$. Here $\alpha$ and $\beta$ are given physical constants. It is shown that the solution of $\psi_{x x}+\psi_{y y}-k^{2} \psi=0$ is subject to the same boundary conditions as may be formulated as a Wiener-Hopf integral equation and its solution may be given in integral form. From this the behavior of $\psi(x, 0)$ may be determined for $|x| \rightarrow \infty$ or $\infty$. It is further shown that $\psi(x, y)$ considered as a function of parameter $k$ is simply $\phi(x, y)$ when $|k| \rightarrow 0$. This problem has been recently considered by E. L. Buell who solved the problem by other methods (Journal of Mathematics and 
Physics vol. 26, (1948) p. 223). The mathematical problem arose in the study of the effect of a semi-infinite elastic stiffener on the boundary of a semi-infinite thin plate. (Received March 2, 1949.)

\section{C. A. Truesdell: The kinematics of vorticity. II.}

The kinematic foundation of the Lagrange-Cauchy velocity-potential theorem is examined in detail. The proofs of Lagrange, Cauchy, Power, Stokes, Kelvin, and St. Venant are examined in the light of the criticisms of Poisson, Stokes, Boussinesq, and Duhem. The Cauchy, Stokes and Kelvin proofs do not require analyticity, but apply only to circulation-preserving motions. The Lagrange, Power, and St. Venant proofs apply to a more general class of motions, but require analyticity. The former yield the true velocity-potential theorem. The latter yield theorems which when applied to certain types of motions which are not circulation-preserving prove that those motions cannot be represented altogether by analytic functions of the time. (Received February 18, 1949.)

375t. A. H. Van Tuyl: On the evaluation of some definite integrals which occur in hydrodynamics and elasticity.

Integrals of the types $I_{1}=\int_{0}^{\infty} e^{-a t} J_{n}(b t) J_{n}(c t) t^{m} d t$ and $I_{2}=\int_{0}^{\infty} e^{-a t} J_{n-1}(b t) J_{n}(c t) t^{m+1} d t$, $a \geqq 0,2 n+m \geqq 0$, occur in potential theory, hydrodynamics, and elasticity for $n=1$, $2, m=0,-1$ in $I_{1}$, and $m=-1,-2$ in $I_{2}$ (A. Weinstein, Quarterly of Applied Mathematics vol. 5 (1948) pp. 429-444, and Courant Anniversary Volume, 1948, pp. 451460 ). It is shown that for every integer $n \geqq 0$ and all permissible positive and negative integers $m, I_{1}$ and $I_{2}$ can be expressed in terms of elliptic integrals. Both complete and incomplete elliptic integrals of the first and second kinds are present for $m<0$, while for $m \geqq 0$, only complete elliptic integrals occur. The case $m \geqq 0$ has been known implicitly (G. N. Watson, Bessel functions, p. 389, and W. M. Hicks, Philos. Trans. Roy. Soc. London Ser. A vol. 172 (1881) pp. 609-652), but the results involving the incomplete elliptic integrals are new. Further, recurrence relations are obtained by means of which every $I_{1}$ and $I_{2}$ for $m<0$ can be expressed in terms of the integrals $\int_{0}^{\infty} e^{-a t} J_{\mu}(b t) J_{\mu}(c t) t^{m} d t$ and $\int_{0}^{\infty} e^{-a t} J_{n}(b t) J_{n}(c t) d t$, where $\mu=[(1-m) / 2]$. Numerical calculations have been made using the preceding results, and are applied to the problems mentioned above. (Received March 4, 1949.)

\section{Alexander Weinstein: On the transonic flow around a wedge.}

Using the results previously obtained in the paper Generalized potential theory (A. Weinstein, Trans. Amer. Math. Soc. vol. 63 (1948) pp. 342-354) an explicit solution involving Weber-Schafheitlin integrals is given of the Tricomi equation $3 y\left(\psi_{x x}+\psi_{y y}\right)$ $+\psi_{y}=0$ in the domain lying above the characteristic $x^{2}+(4 / 9) y^{8}=0$ and bounded by the verticals $x=0$ and $x=1$. The solution vanishes on the verticals $x=0, y>1$ and $x=1, y>-(3 / 2)^{2 / 3}$ and is equal to one on the segment $x=0,0 \leqq y<1 . \psi(x, y)$ represents, in a distorted hodograph plane, the stream function of a transonic flow around a wedge in a channel which is rectilinear in the subsonic part of the flow. (Received March 18, 1949.)

\section{GEOMETRY}

377t. Edward Kasner and John DeCicco: Physical curves in space of $n$ dimensions.

A system $S_{k}$ of $\infty^{2 n-1}$ curves in a given positional field of force in space of $n$ dimensions consists of curves along which a constrained motion is possible such that 
the osculating plane at each point contains the force vector $F$, and the pressure $P$ along the principal normal to the curve is proportional to the normal component $N$ of $F$. Consider the $\infty^{1}$ curves of a system $S_{k}$ passing through a given point in a given direction. The initial osculating plane and three-flat are fixed. The osculating $m$-flats, for $m=4,5, \cdots, n-1$, vary and form one-parameter families. The first curvature $k_{1}$ varies inversely as the square of the speed $v$. The rate of variation $d \rho_{1} / d s$ of the first radius of curvature $\rho_{1}$ is a linear integral function of $\rho_{1}$. The second curvature $k_{2}$ is constant. The remaining curvatures $k_{3}, k_{4}, \cdots, k_{n-1}$ are algebraically dependent on $k_{1}$. The locus of the centers of the osculating $m$-spheres for $m=3,4, \cdots, n-1$ is an algebraic curve. For $m=2$, the locus of the centers of the osculating spherical surfaces is a straight line just as in the case for $n=3$. The interrelationships between the rest trajectories and the lines of force are also discussed. (Received February 15, 1949.)

\section{8t. Edward Kasner and John DeCicco: Scale curves in general surface mapping.}

Let a point transformation $T$ be defined between two surfaces $S$ and $S_{1}$ of Euclidean space such that points correspond if they are represented by the same curvilinear coordinates $(x, y)$. The scale function $\sigma=d s / d s_{1}$ is the ratio of the differentials of the arc lengths. Only nonconformal maps are studied so that $\sigma$ is dependent on $d y / d x$ explicitly. The curves $\sigma=$ const. are called the scale curves of the mapping $T$. These may be represented on $S$ or $S_{1}$. There are $\infty^{2}$ scale curves. At a point $(x, y)$ there are five inflectional directions and two cuspidal directions for the scale curves. The cuspidal directions on $S$ and $S_{1}$ are always orthogonal, and they coincide with the characteristic directions on $S$ and $S_{1}$. The central locus corresponding to a given point $(x, y)$ is an algebraic curve of the fifth degree. The associated scale quartic is studied. (Received December 27, 1948.)

\section{9t. Edward Kasner and Don Mittleman: Second order differ- ential equations of rank 2.}

Second order differential equations of rank 2 are of the form $A_{0} y^{\prime \prime 2}+\left(B_{0}+B_{1} y^{\prime}\right.$ $\left.+B_{2} y^{\prime 2}+B_{3} y^{\prime 3}\right) y^{\prime \prime}+\left(C_{0}+C_{1} y^{\prime}+C_{2} y^{\prime 2}+C_{3} y^{\prime 3}+C_{4} y^{\prime 4}+C_{5} y^{\prime 5}+C_{6} y^{\prime 8}\right)=0$ where the coefficients are arbitrary functions of $x, y$. Kasner had shown that under an arbitrary point transformation an equation of rank 2 is transformed into an equation of rank 2. At a fixed point, the locus of the centers of the osculating circles of the integral curves of the equation lie on a special sextic, which may degenerate, in a nontrivial way, to a special quartic or a conic. Differential equations of rank 2 arise in the study of scale curves, certain two-parameter families of curves which are quadratic in the parameters, as the images of the $\infty^{2}$ circles of unit radius under an arbitrary point transformation, as extremals of the variational problem $\delta \int[\nu(x y) d s+(A d x+B d y)]=0$, in the study of the motion of an electric particle in a static electromagnetic field, and in the restricted problem of three bodies in the plane. (Received March 8,1949.)

\section{0t. Wouter van der Kulk: The univalent algebraic transformations of the projective plane.}

A univalent function on the projective plane $P$ onto $P$ whose graph is an algebraic variety in $P \times P$ over a field $\Delta$ is called a univalent algebraic transformation of $P$ over $\Delta$ (uat). If $\Delta$ has characteristic 0 then it is well known that the group $G$ of uat's coincides with the group of projective transformations of $P$. This is no longer true if the characteristic of $\Delta$ is $p(p>0)$. In that case it is possible to construct a set of generators 
of $G$, called semi-projective transformations of $P$ over $\Delta$ (spt). A spt can be characterized as a uat which maps either every straight line in $P$ or every straight line of some linear pencil in $P$ onto a straight line in $P$. The uniqueness of the representation of a uat as product of spt's is investigated. The proofs of the preceding results are based on the theory of algebraic curves over a perfect field (cf. W. L. Chow, Die geometrische Theorie der algebraischen Funktionen filr beliebige vollkommene Körper, Math. Ann. vol. 114 (1937)). (Received March 14, 1949.)

\section{1t. Jack Levine: Classification of two-dimensional affinely con- nected spaces admitting groups of collineations.}

By using Lie's classification of continuous groups in two variables (Theorie der Transformationsgruppen, vol. 3, pp. 71-73) all two dimensional affinely connected spaces $\left(A_{2}\right)$ are obtained which admit groups $G_{r}$ of projective collineations. It is found that all $G_{r}$ are possible for $1 \leqq r \leqq 8$, with the exception of $r=7$. There are 25 types of non-equivalent groups represented. The $A_{2}$ 's corresponding to 22 of the groups are projectively flat. The 3 groups corresponding to non-projectively flat spaces are $[p]$, $[p, x p+y q],[p, 2 x p+y q, x p+x y q]$. As special cases, the $A_{2}$ admitting affine and translatory collineations are found. The largest affine collineations group is a $G_{4}$ if the $A_{2}$ is not flat. The Riemannian $V_{\mathbf{2}}$ admitting projective and affine collineations are also determined. The only $V_{2}$ of non-constant curvature admitting a $G_{r}(r>1)$ of affine collineations is $d s^{2}=y^{m}\left(a d x^{2}+2 d x d y+b d y^{2}\right)$ with $a, b, m$ constants, and $m \neq-2$. The associated group is $[p, x p+y q]$, and it contains a one-parameter subgroup of motions. (Received March 7, 1949.)

\section{2t. Don Mittleman: Velocity families of rank 2.}

A two-parameter family of plane curves is called a velocity family of rank 2 if, at each point $p$, the centers of the osculating circles of those members of the family which pass through $p$ lie on a conic for which $p$ is a focus. An arbitrary nonsingular point transformation $T$ will convert a velocity family into a velocity family if and only if $T$ is conformal (either direct or reverse). Velocity families of rank 2 arise in the study of the plane motion of an electric particle in a static electro-magnetic field, as the conformal images of $\Gamma^{*}$ curves (a two-parameter family whose curvature is a point function), as extremals of a variational problem derived from Hamilton's principle for non-conservative fields, and in the motion of a particle in a positional field of force in a rotating plane. This last case includes the restricted problem of three bodies in the plane. These various problems are not all equivalent under a conformal transformation. The conformal covariants of a velocity system are determined. Thus, problems which give rise to velocity families of rank 2 may be classified as being conformally equivalent or distinct. (Received March 8, 1949.)

\section{3t. W. G. Wolfgang. A representation of complex-two space.}

Define complex-two space $C_{8}$ to be the space whose points may be put into one-toone correspondence with the set of all pairs $(A, B)$, where $A$ and $B$ are complex numbers. The purpose of the author is to give a construction of a representation of space $C_{2}$ in Euclidean three-space $R_{3}$. From the geometric construction the representation is defined by means of the mapping: $f: C_{2} \rightarrow R_{3}$ where $f(X, Y)=(x, y, z)$ is defined by $x=x^{\prime}, y=x^{\prime \prime}+y^{\prime}, z=y^{\prime \prime}$, where $X=x^{\prime}+x^{\prime \prime} i, Y=y^{\prime}+y^{\prime \prime} i$. This mapping is not one-to-one and some time is spent in characterizing the points of $C_{2}$ which map into the same point of $R_{8}$. By means of this mapping the representations of several of the 
familiar "curves" of analytic geometry as configurations in this space are discussed and constructed. A completely general analytic definition of the representation, of which the one previously presented is a specific form, is also developed. (Received March 17, 1949.)

384. W. G. Wolfgang. Metric considerations in complex-two space.

Define complex-two space to be the space whose points may be put into one-to-one correspondence with the set of all pairs $(A, B)$, where $A$ and $B$ are complex numbers. The usual definition of a distance function, $\rho(P, Q)=\left(\left(X_{1}-X_{2}\right)^{2}+\left(Y_{1}-Y_{2}\right)^{2}\right)^{1 / 2}$, $P:\left(X_{1}, Y_{1}\right), Q:\left(X_{2}, Y_{2}\right)$, defined in this space is not a true metric. The purpose of the author is to show that the space is metrizable in the usual sense and that the distance function $\rho(P, Q)=\left(\left|X_{1}-X_{2}\right|^{2}+Y_{1}-\left.Y_{2}\right|^{2}\right)^{1 / 2}$ is a true metric for the space. (Received March 17, 1949.)

\section{LOGIC AND Foundations}

385. Ilse L. Novak: $A$ construction of models for consistent systems of axioms.

The methods in the author's paper The relative consistency of von Neumann's and Zermelo's axioms for set theory (Bull. Amer. Math. Soc. abstract 55-3-201)are here generalized to apply to any consistent system $S$ which (i) contains a finite number of primitive functors, (ii) is based on a finite number of axioms and axiom-schemata and (iii) has rules of deduction based on first order functional calculus. The author describes the construction of a model of $S$ in the syntax of $S$, based only on the hypothesis that $S$ is consistent and assumptions equivalent to the 5 Peano axioms for arithmetic. This construction makes possible a purely arithmetical proof of the Skolem-Loewenheim Theorem. A model of $S$ obtained by this method can be enlarged to one of a larger system $S^{\prime}$ related to $S$ as the von Neumann-Bernays-Gödel system is to the Zermelo-Fraenkel system. The methods described therefore also provide a purely arithmetical proof of the relative consistency of $S$ and $S^{\prime}$. (Received April 6, 1949.)

\section{Statistics AND Probability}

386. K. L. Chung: Fluctuations of sums of independent random variables.

Let $S_{n}$ be the sum of $n$ independent random variables having the same non-lattice distribution $F(x)$ with mean 0 , variance 1 , and finite third absolute moment. Let $c$ be any real number and $T_{n}$ denote the number of $k$ 's not exceeding $n$ for which $S_{k}>c$, $S_{k+1}<c$. Then $\lim P\left(T_{n} \leqq 2^{-1} \beta_{1} n^{1 / 2} x\right)=(2 / \pi)^{1 / 2} \int_{0}^{x} e^{-y^{2} / 2} d y$ where $\beta_{1}=\int_{-\infty}^{\infty}|x| d F(x)$. There is a corresponding theorem for a lattice distribution, when the factor $2^{-1} \beta_{1}$ in the above formula is replaced by a slightly different number. In particular if $c=0$, the limiting distribution of the number of changes of sign of $S_{n}$ is obtained. The method of proof is that of convergence of moments. (Received March 11, 1949.)

\section{D. A. Darling: A theorem on stable distributions.}

Let $X$ and $Y$ be positive independent random variables distributed according to a stable law with exponent $\alpha$ so that $E(\exp i z x)=\exp \left(-c_{1}(i z)^{\alpha}\right), E(\exp i z y)$ $=\exp \left(-c_{2}(i z)^{\alpha}\right), 0<\alpha<1, c_{1}>0, x_{2}>0, z>0, i^{\alpha}=\exp (\pi i \alpha / 2)$. Then $\operatorname{Pr}\left\{(1+x / y)^{-1}\right.$ $<\beta\}=F(\beta)=1 / 2 \alpha-(1 / \pi \alpha) \tan ^{-1}\left\{\left(\beta^{-1}(1-\beta)\right)^{\alpha}+c \cos \pi \alpha / c \sin \pi \alpha\right\}, 0 \leqq \beta \leqq 1$, 
$c=c_{1} / c_{2}$. This expression gives, under certain circumstances, the limiting distribution for the proportion of time a Markoffian variable spends in a given state. For example, when $\alpha=1 / 2, c=1, F(\beta)=(2 / \pi) \sin ^{-1} \beta^{1 / 2}$. (Received March 17, 1949.)

\section{D. A. Darling: Sums of symmetrical random variables.}

Let $X_{1}, X_{2}, \cdots$, be independent, symmetrical, identically distributed random variables with a continuous distribution, but otherwise arbitrary. The principal result is that the probability that the first $n$ partial sums are positive is independent of the distribution of the $X$ 's. Specifically, letting $S_{n}=X_{1}+X_{2} \cdots+X_{n}, S_{0}=0$, $\operatorname{Pr}\left\{s_{1}>0, s_{2}>0, \cdots, s_{n}>0\right\}=u_{n}=2^{-2 n} C_{2 n, n}$. From this fact several theorems follow: (1) Let $M_{n}$ be that value of $j$ for which $S_{j}$ attains its maximum $(j=0,1, \cdots, n)$, then $\operatorname{Pr}\left\{M_{n}=k\right\}=u_{k} u_{n-k}$. (2) Let $L_{n}$ be the smallest value of $j$ for which $S_{i+1}$, $S_{j+2}, \cdots, S_{n}$ are of constant sign $(j=1,2, \cdots, n-1), L_{n}=0$ if all $S_{j}$ are $\leqq 0$, and $L_{n}=n$ if all $S_{j}$ are $\geqq 0$, then $\operatorname{Pr}\left\{L_{n}=k\right\} \backsim u_{k} u_{n-k}$, for large $n, k$. (3) Let $N_{n}$ be the number of positive $S_{j}$ for $j=1,2, \cdots, n$, then $\operatorname{Pr}\left\{N_{n}=k\right\}=u_{k} u_{n-k}$. Limiting expressions for these theorems are as follows: $\lim _{n \rightarrow \infty} \operatorname{Pr}\left\{T_{n}<n x\right\}=2 \pi^{-1} \sin ^{-1} x^{1 / 2}$ where $T_{n}$ is any one of the three random variables $M_{n}, L_{n}$ or $N_{n}$. The limiting distribution for $N_{n}$ is in a certain way a generalization of an earlier result of Erdös and Kac (Bull. Amer. Math. Soc. vol. 53 (1947) pp. 1101-1120). (Received April 4, 1949.)

389t. Mark Kac and Harry Pollard: The distribution of the maximum of sums of independent random variables.

Let $X_{1}, X_{2}, \cdots$ be independent random variables each having the same Cauchy density function $\pi^{-1}\left(1+x^{2}\right)^{-1}$. Let $s_{k}=X_{1}+\cdots+X_{k}$ and consider the probability $P_{n}(a)$ that $\left|s_{n}\right|<a n$ for $k=1,2, \cdots, n$. In this paper the limit of $P_{n}(a)$, as $n \rightarrow \infty$, is calculated in terms of the eigenvalues and eigenfunctions of the kernel $K(x, y)$ $=4^{-1}\left\{\log \left(1-x y+\left(1-x^{2}\right)^{1 / 2}\left(1-y^{2}\right)^{1 / 2}\right)-\log \left(1-x y-\left(1-x^{2}\right)^{1 / 2}\left(1-y^{2}\right)^{1 / 2}\right\}, \quad 1 \leqq x\right.$, $y \leqq 1$. For the sake of comparison the method is also applied to the case of normally distributed $X_{j}$ and the known answer is rederived. (Received February 14,1949.)

\section{TOPOLOGY}

\section{A. L. Blakers and W. S. Massey: Homotopy groups of triads.}

A triad $(X ; A, B)$ consists of a topological space $X$ and two subspaces $A$ and $B$ for which $A \cap B \neq 0$. Let $E^{n}$ be an $n$-cell, $E_{+}^{n-1}$ and $E_{-}^{n-1}$ the upper and lower "hemispheres" of its boundary sphere $S^{n-1}, p_{0} \in E_{+}^{n-1} \cap E_{-}^{n-1}$, and $x_{0} \in A \cap B$. An addition is defined between homotopy classes of maps $\left(E^{n} ; E_{+}^{n-1}, E_{-}^{n-1}, p_{0}\right) \rightarrow\left(X ; A, B, x_{0}\right)$ in such a way that these homotopy classes form a group $\pi_{n}(X ; A, B)(n \geqq 3)$. These groups fit into two exact sequences each similar to the homotopy sequence of a pair. In case $X=A \cup_{B}$, the triad homotopy groups $\pi_{n}(X ; A, B)$ are a measure of the amount by which the triad $(X ; A, B)$ fails to satisfy the excision axiom (see $\mathrm{R}$. $\mathrm{H}$. Fox, Ann. of Math. vol. 49 (1948) pp. 484-485). The triad homotopy groups are used to give a new interpretation and proof of the Freudenthal "Einhängung" theorems (Compositio Mathematica vol. 5 (1937) p. 299), and to study the effect produced on the homotopy groups of a complex when a new cell is adjoined. (Received March 17, 1949.)

\section{W. A. Blankinship: Generalization of a construction by Antoine.}

In $1921 \mathrm{~L}$. Antoine constructed a compact, zero-dimensional set in $R^{8}$ whose complement is not simply connected. In the present paper Antoine's construction is 
generalized to give such a point set in Euclidean space of arbitrary dimension. As a corollary to the construction, one is also enabled to construct a set in $R^{n}, n \geqq 3$, homeomorphic to a $q$-cell, $0<q \leqq n$, whose complement is not simply connected. It is further shown that the complement of any compact set in Hilbert space is contractible, and hence sets of the type above mentioned cannot exist in Hilbert space. (Received March 14, 1949.)

\section{J. H. Blau: The space of measures on a given set.}

Let $R$ be an abstract set with a class of subsets called "open," satisfying only the condition that $R$ is open. A measure is a Caratheodory outer measure which is regular with respect to the open sets, and for which open sets are measurable. A neighborhood topology is introduced in the set $M$ of all measures, under which $M$ becomes a $T_{1}$ space. This topology specializes to weak convergence under certain conditions. Suitable restrictions on the "open" sets of $R$ imply topological properties of $M$. Necessary and sufficient conditions are found under which $M$ is separable, Hausdorff, bicompact, and metrizable. (Received March 17, 1949.)

\section{3t. D. G. Bourgin and Abraham Charnes: On some normed rings.}

The introduction of multiplications into Banach spaces and the structures of the topological algebras so obtained is studied from a Banach space standpoint. Semisimplicity, existence and equivalence of multiplications are characterized in terms of Banach isomorphisms. In another direction the ring of continuous functions $C(S)$ on a completely regular space $S$ wtih $F$ metric is studied. If $T$ is an isometry of $C\left(S_{1}\right)$ on $C\left(S_{2}\right)$ and the maximum free ideals of $C\left(S_{i}\right)$ are not closed, and if pointwise convergence implies convergence of the $T$ maps, then $S_{1}$ and $S_{\mathbf{2}}$ are homeomorphic. (Received April 11, 1949.)

\section{E. E. Floyd: Some characterizations of interior maps.}

Several characterizations of light interior maps are given, each of which is one. dimensional in nature. Two results are the following. Let $X$ and $Y$ be compacta, and let $f$ be a light map of $X$ onto $Y$. If $X$ is locally connected and connected, then $f$ is interior if and only if for each region $V$ in $Y$ and each component $U$ of $f^{-1}(V)$, $f^{*}: H^{1}(\bar{V}, \bar{V}-V) \rightarrow H^{1}(\bar{U}, \bar{U}-U)$ is an isomorphism into, where $H^{1}(X, A)$ denotes the one-dimensional Cech cohomology group of $X$ relative to $A$ with integer coefficients. Also $f$ is interior if and only if for each map $h: I \rightarrow Y, I$ the unit interval, and for each $x \in f^{-1} h(0)$ there exists a map $g: I \rightarrow X$ with $f g=h$ and $g(0)=x$. (Received March 10, 1949.)

\section{R. H. Fox: The order of the homology group of a cyclic covering of a knot.}

Let $S_{g}$ denote the $g$-fold cyclic covering of spherical 3-space $S$, branched over a tame simple closed curve $k$. It is known (Alexander, Trans. Amer. Math. Soc. vol. 30 (1928) pp. 275-306 or Reidemeister, Knotentheorie) that the first homology group $H_{\theta}$ of $S_{o}$ is determined by the positive integer $g$ and the fundamental group of $S-k$, and is therefore an invariant of the knot-type of $k$. It is shown that the order of the group $H_{\theta}$ is determined by $g$ and Alexander's polynomial $\Delta(t)$ alone; in fact the order of $H_{\theta}$ is equal to the resolvent of the two polynomials $t^{0}-1$ and $\Delta(t)$. (Received March 17, 1949.) 
396. C. N. Reynolds: On the problem of coloring maps in four colors.

This is an application of recent papers by the author on a calculus of topologically defined finite differences. (Received February 23, 1949.)

\section{7t. G. T. Whyburn: On open and closed mappings.}

If $A$ and $B$ are Hausdorff spaces, a mapping $f(A)=B$ is quasi-compact if the image of every open (or closed) inverse set in $A$ is open (closed) and is compact if the inverse of every compact set in $B$ is compact. Any two factorizations $f=h \phi$, where $h$ is 1-1 and $\phi$ is quasi-compact, are topologically equivalent as are also any two monotonelight factorizations of $f$ whose monotone factors are quasi-compact. A mapping $f$ is open (closed) if and only if it is quasi-compact and generates a l.s.c. (u.s.c.) decomposition of $A$. In order that $f$ generate a (1) l.s.c. (2) u.s.c. (3) continuous, decomposition of $A$ it is necessary and sufficient that it factor into the topologically unique form $f=h \phi$ where $h$ is a 1-1 mapping and $\phi$ is (1) open (2) closed or (3) both open and closed, respectively. If $f$ is closed it factors in the form $f=h r g$ with $g$ compact, $r$ a closed retraction and $h$ a homeomorphism. If $A$ is locally compact separable and metric and components of the sets $f^{-1}(y), y \in B$, are compact, $f$ has a topologically unique monotone-light factorization with closed monotone factor. Normality and perfect separability are invariant under closed mappings. (Received March 17, 1948.)

\section{T. R. HollCROFT, Associate Secretary}

